\title{
Quartäre Klimaphasen und Subrosion als Faktoren der Bildung von Kies-Terrassen im südwestlichen Harzvorland
}

\author{
Werner Ricken *)
}

Landform evolution, gravel terrace, fluviatile erosion, aggradation, fluvial sedimentation, loess soil, paleosol, section, longitudinal profil, biostratigraphy, influence, paleoclimatology, subrosion, Elster Glacial, Warthe Glacial, Weichsel Glacial. Southwestern Harz Mountains, Southwestern Harz Foreland, Schwarzfeld Hattorf Lonau Region, Niedersachsen, TK 25 Nr.: 4327, 4328

Kurzfassung: Aufbau und Verbreitung der Kieskörper der Harzflüsse Sieber und Oder werden beschrieben, sowie verschiedene Erosionsterrassen ohne eigenen Kieskörper. Das Alter der Kiese und der Erosionsstufen kann mit den sich teilweise verzahnenden und überlagernden Lössen und deren Böden und auf biostratigraphischem Wege bestimmt bzw. eingeengt werden. Hierbei ergeben sich folgende Alter: Oberterrasse mindestens Elster, Altere Mittelterrasse mindestens Drenthe, Mittelterrasse mindestens Warthe, Aue-Berg-Terrasse II Altweichsel, Ältere Niederterrasse Altweichsel bis Jungweichsel. Der Komplex der Weichsel-zeitlichen Kieskörper setzt sich in Subrosionsgebieten aus einer ersten bedeutenden Akkumulation im Altweichsel (Aue-Berg-Terrasse II und unterer Kieskörper der Alteren Niederterrasse) und neben mehreren kleineren Erosions- und Akkumulationsphasen aus einem Kieskörper der Jungweichsel-Zeit zusammen (oberer Kieskörper der Älteren Niederterrasse).

Aus der Verzahnung mit den Lössen und ihren Derivaten folgt, daß der größte Teil der Terrassen-Kiese im Untersuchungsgebiet unter feucht-kaltem Solifluktionsklima gebildet worden ist (Aue-Berg-Terrasse II, unterer Kieskörper der Alteren Niederterrasse, vielleicht auch Oberterrasse), jedoch hat es auch bedeutende Akkumulationen unter trocken-kalten Klimaverhältnissen gegeben (oberer Kieskörper der Alteren Niederterrasse, vermutlich auch Mittelterrasse). Erosionsphasen unter kaltem Klima - teilweise Tundrenklima - wurden festgestellt (Beginn von Mittel-, Jungund Spätweichsel, vermutlich auch nach der Sedimentation der Älteren Mittelterrasse).

Eine Bifurkation der Sieber nach Verlassen des Harzes ist für fast alle Akkumulationsphasen belegt. In den Eintiefungsphasen während des Eem-Interglazials und vermutlich auch vor der Akkumulation der Mittelterrasse wurde jedoch, wie auch heute, der konsequent verlaufende Sieberarm aufgegeben (Aue-Tal) und nur der Talabschnitt im Zechstein benutzt (Hördener Tal). Das Hördener Tal ist in den Interglazialen jeweils aufgrund von Subrosions- und Erosionsprozessen übertieft worden.

Es gibt zwei Typen von fluviatil geprägten Subrosionssenken. Die subrosive Absenkung kann überwiegend synchron mit der Kiessedimentation verlaufen (synsedimentäre Subrosion), oder sie kann überwiegend zeitgleich mit der Fluß-Erosion wirken (synerosive Subrosion). Synsedimentäre Subrosion hat im Altweichsel im Pöhlder Becken die Bildung eines über $50 \mathrm{~m}$ mächtigen Kieslagers verursacht. Synerosive Subrosion hat im Eem-Interglazial, wie auch heute westlich von Herzberg, eine Übertiefung des Siebertales ausgelöst. Im nachfolgenden Weichsel-Glazial ist dann in dem übertieften Talabschnitt der übernormal mächtige Kieskörper der Alteren Niederterrasse sedimentiert worden.

\section{[Quarternary Climatic Phases and Subrosion as Factors for the Origin of Gravel Terraces in the Southwestern Foreland of the Harz-Mountains]}

A bs tract: Structure and distribution of gravel bodies of the rivers Sieber and Oder are described. Furthermore other terraces exist which are formed by erosion and thus do not posess an own gravel body. The minimum age of the gravel layers, climatic variations, and solifluction intensity are derived from the stratigraphical study of the loess cover and by paleobotanical determinations. The resulting data are as follows: Upper Terrace Elster or older, Upper Middle Terrace probably Drenthe, Middle Terrace Warthe or older, Mount Aue Terrace II Old Weichselian, Older Lower Terrace Old Weichselian to Young Weichselian. The complex Weichselian

*) Anschrift des Autors: Dipl.-Geologe W. Ricken, Geol.-Paläont.-Institut, Sigwartstr. 10, 7400 Tübingen. 
gravel layer was formed by a first strong phase of accumulation in the Old Weichselian (Mount Aue Terrace II, lower gravel body of the Older Lower Terrace). After some phases of erosion and sedimentation, a minor accumulation took place in the Young Weichselian.

The main phases of gravel sedimentation happened under cold and wet climatic conditions, due to high rates of solifluction (Mount Aue Terrace II, lower gravel body of the Older Lower Terrace, probably Upper Terrace). Nevertheless, gravel sedimentation also occured in a dry cold climate with loess sedimentation (upper gravel body of the Older Lower Terrace, probably Middle Terrace). Erosion took place during tundra climate and at the transition glacial/interglacial (beginning of the Middle Weichselian, of the Young Weichselian and of the Late Weichselian), while in recent days the interglacial erosion is minute.

Bifurcation of the river Sieber happened at almost any stages of gravel accumulation. However, in periods of erosion one tributary dryed up and the river only used the tributary in the Zechstein-outcrop parallel to the Harz Mountains. This happened in the Eemian as well as it does in recent days, because the tributary in the Zechstein-outcrop was and is strongly deepened by combined process of subrosion and erosion and thus preferred.

Subrosion of Zechstein-gypsum caused two fundamental types of subrosion basins, filled with gravels. 1) Subrosion and land sink had been synchronous with the gravel accumulation leading to badly stratified gravel basins (Basin of Pöhlde, Main-Anhydrite). 2) Subrosion and land sink had been synchronous with the interglacial erosion. In this case the vally had been deepened very strongly and retrogressive erosion formed canyons in the greywacke of the Harz Mountains. In the following glacial period gravel accumulation filled up the valley and by this a huge stratified gravel body originated (lower gravel body of the Older Lower Terrace).

\section{Einleitung}

Pleistozäne Flußkiese erreichen am südlichen Rand des Harzes bis zu $100 \mathrm{~m}$ Mächtigkeit und zwar dort, wo die Harz-Flüsse den Zechstein queren und ihre Kiese in Subrosionssenken oder übertieften Flußrinnen ablagern bzw. ablagerten. Im W und E verzahnen sich die Kiese des S-Harzes teilweise mit Moränen der nordischen Vereisung, jedoch ist bisher nur eine unvollkommene Parallelisierung der Flußkiese mit ehemaligen Gletschervorstößen gelungen. Deshalb kommt den Lössen und Paläoböden, die die Kiese überlagern und sich mit ihnen teilweise verzahnen, für die stratigraphische Zuordnung der Kieskörper die größte Bedeutung zu.

Die vorliegende Arbeit behandelt nur einen kleinen Ausschnitt aus dem Kies-Gürtel des S-Harzes, das südliche Flußgebiet der Sieber und das südlich daran anschließende Pöhlder Becken im Odertal (Abb. 1). Der präquartäre Untergrund wird vom Variszikum des Harzes, Gipsen und Dolomiten des Zechsteins und vom Buntsandstein gebildet. Flußkiese bedecken in ca. $5 \mathrm{~m}$ bis $15 \mathrm{~m}$ Mächtigkeit Harz-Paläozoikum und Buntsandstein und erreichen nur im Zechstein höhere Mächtigkeiten. Mit neueren, z. T. unpublizierten Arbeiten liegt eine Fülle von Daten vor (Jordan 1976, 1979; Piehler 1969; Ricken 1973, 1980; Thiem 1972; VLADI 1976), so daß sich das geologische Bild allmählich abzurunden beginnt. Das südliche Harzvorland bietet die Möglichkeit, fluviatile Erosions- und Akkumulationsphasen des Pleistozäns klimatisch, genetisch und zeitlich einzuordnen. Ferner liefern die hier beispielhaft entwickelten Subrosionssenken im Zusammenhang mit der Flußgeschichte Daten zur Entstehung von mächtigen Kies-Lagern.

Drei Aspekte der Quartärgeologie des südwestlichen Harzvorlandes sollen in dieser Arbeit besonders hervorgerufen werden:

1. Von den drei großen, in S-Niedersachsen verbreiteten Kieskörpern sind die Ober- und Mittelterrasse und teilweise auch die Niederterrasse bis ca. $330 \mathrm{~m}$ Geländehöhe von Lössen unterschiedlichen Alters bedeckt, die eine Mächtigkeit bis zu $15 \mathrm{~m}$ erreichen können. In 150 Bohrungen und Aufschlüssen aufgenommen, erwies sich der Löß durch die darin enthaltenen Paläoböden als stratifizierbar (RICKEN, im Druck). Die Lößderivate liefern neben einem Mindestalter der Kies-Terrassen auch 


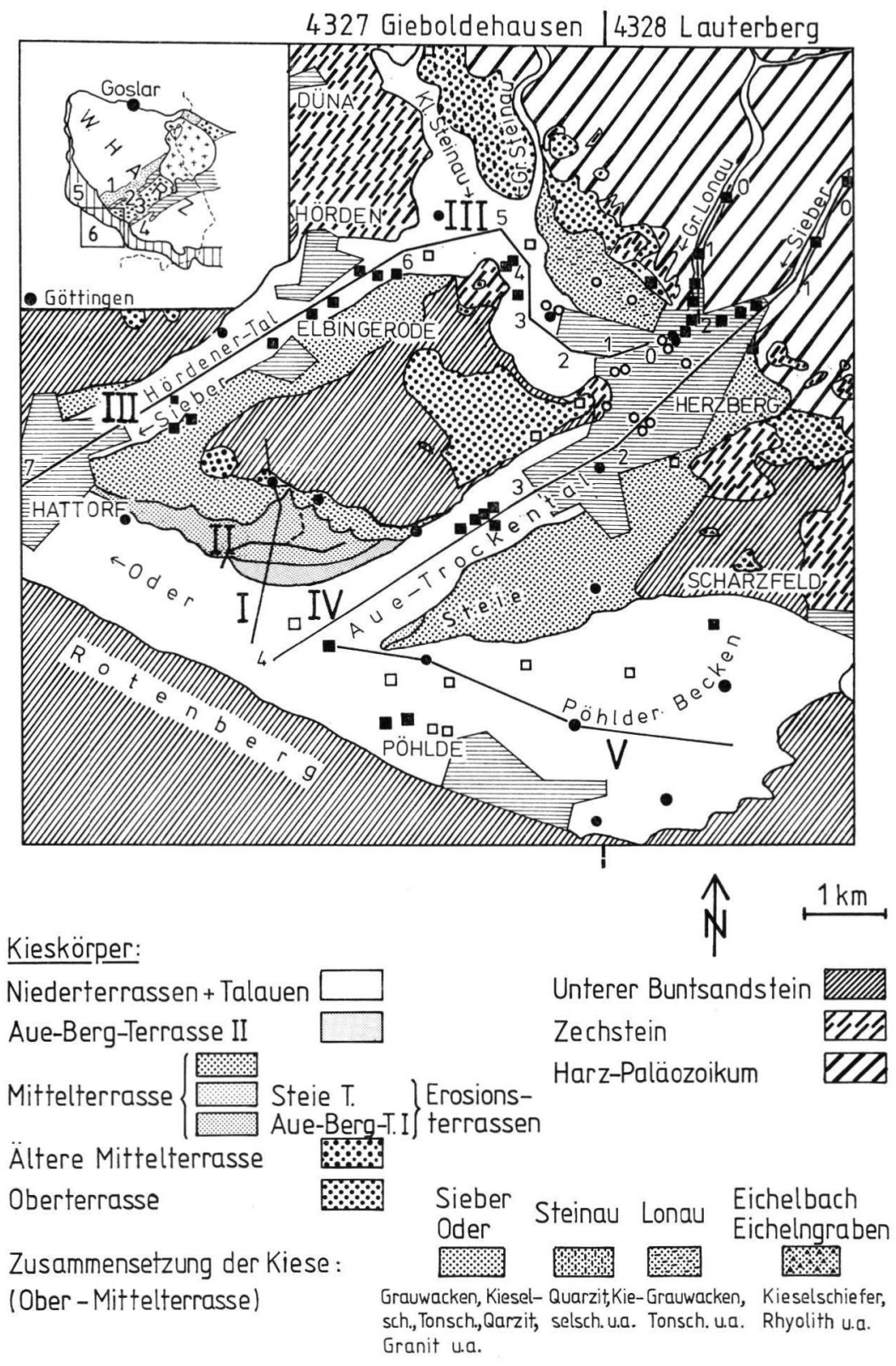

Abb. 1: Geologische Übersicht. Profile I bis V s. Abb. 2, 7, 8, 9.

- $\square$ Aufschluß, Quartärbasis erreicht bzw. nicht erreicht. $\bigcirc$ Bohrung, Quartärbasis erreicht bzw. nicht erreicht. - Nebenkarte: 1 Acker-Hörre-Quarzit (U. Karbon). 2 Tonschiefer und Kieselschiefer des Lonauer Sattels (Ob. Devon). 3 Sieber-Grauwacke (U. Karbon). 4 Tonschiefer und Kieselschiefer des Herzberger Sattels (Devon). 5 Anhydrit und Dolomit (Zechstein). 6 Tonstein und Sandstein (Unterer Buntsandstein). 
Tab. 1: Boden und Terrassenstratigraphie des südwestlichen Harzvorlandes, nach Ricken (im Druck). Begriffe Alt-Mittel- und Jungweichsel nach Rohdenburg \& MeYer (1966).

\begin{tabular}{|c|c|c|c|c|}
\hline Zeit & Paläoböden & Kieskörper & $\begin{array}{l}\text { Erosions- } \\
\text { terrassen }\end{array}$ & $\begin{array}{l}\text { Verwitterungsgrad } \\
\text { der Grauwacken- } \\
\text { Komponenten } \\
\text { nach MunSELL (1954) }\end{array}$ \\
\hline $\begin{array}{l}\text { ca. O bis } \\
\text { 18. Jahrh. }\end{array}$ & & $\begin{array}{l}\text { Jüngere } \\
\text { Talaue }\end{array}$ & & \\
\hline $\begin{array}{l}\text { jüngeres } \\
\text { Holozän }\end{array}$ & & Altere Talaue & & \\
\hline Spätweichsel & $?$ & & $\begin{array}{l}\text { Jüngere Nie- } \\
\text { derterrasse }\end{array}$ & \\
\hline Jungweichsel & $\begin{array}{l}\text { mehrere } \\
\text { kleinere Bo- } \\
\text { denbildungen }\end{array}$ & $\begin{array}{l}\text { oberer } \\
\text { Kieskörper } \\
\text { d. Älteren } \\
\text { Niederterr. }\end{array}$ & (Markau) & $\begin{array}{l}5 \mathrm{Y} 5 / 1 \text { bis } 10 \mathrm{YR} 4 / 2 \\
\text { grau bis dunkelgrau }\end{array}$ \\
\hline $\begin{array}{c}\text { Stillfried } \\
\text { B } \\
\text { Mittelweichsel }\end{array}$ & $\begin{array}{l}\text { Lohner Boden } \\
\text { Hattorfer } \\
\text { Boden } \\
\text { Herzberger } \\
\text { Boden }\end{array}$ & $\begin{array}{l}\text { Kiessedimen- } \\
\text { tation ? }\end{array}$ & $\begin{array}{l}\text { Steie } \\
\text { Terrasse }\end{array}$ & \\
\hline Altweichsel & $\begin{array}{l}\text { mehrphasige } \\
\text { pseudo- } \\
\text { vergleyte } \\
\text { Parabraun- } \\
\text { erden }\end{array}$ & $\begin{array}{l}\text { Aue-Berg- } \\
\text { Terrasse II, } \\
\text { unterer Kies- } \\
\text { körper der } \\
\text { Alteren Nie- } \\
\text { derterrasse }\end{array}$ & & $\begin{array}{l}5 \mathrm{Y} 6 / 1 \text { bis } 6 / 2 \text { bis } \\
6 / 3 \text { oliv-grau }\end{array}$ \\
\hline Eem & $\begin{array}{l}\text { pseudo- } \\
\text { vergleyte } \\
\text { Parabraun- } \\
\text { erde }\end{array}$ & & $\begin{array}{l}\text { Aue-Berg- } \\
\text { Terrasse I }\end{array}$ & \\
\hline Warthe oder älter & & $\begin{array}{l}\text { Mittel- } \\
\text { terrasse }\end{array}$ & & $\begin{array}{l}5 \mathrm{Y} 6 / 3 \text { bis } 6 / 2 \text { bis } \\
5 / 2 \text { schwach grünlich }\end{array}$ \\
\hline $\begin{array}{l}\text { Drenthe/ } \\
\text { Warthe? }\end{array}$ & $\begin{array}{l}\text { Hördener } \\
\text { Boden- } \\
\text { komplex }\end{array}$ & & & \\
\hline Drenthe oder älter & & $\begin{array}{l}\text { Altere Mittel- } \\
\text { terrasse }\end{array}$ & & \\
\hline $\begin{array}{l}\text { Altsaale oder } \\
\text { Holstein }\end{array}$ & $\begin{array}{l}\text { pseudo- } \\
\text { vergleyte } \\
\text { Parabraun- } \\
\text { erde }\end{array}$ & & & \\
\hline Elster ? & & Oberterrasse & & $\begin{array}{l}2,5 \mathrm{Y} 5 / 4 \text { bis } 6 / 4 \text { bis } \\
6 / 6 \text { schwach oliv- } \\
\text { braun bis grün-gelb }\end{array}$ \\
\hline
\end{tabular}


eine Fülle von paläoklimatischen und landschaftsgenetischen Daten, z. B. Phasen verstärkter Solifluktion. Dies gilt besonders für die Weichsel-zeitlichen Lösse und die mit ihnen korrespondierenden Kieskörper, die in dieser Arbeit bevorzugt behandelt werden. Weitere Kriterien zur zeitlichen Einstufung der oftmals isoliert auftretenden und von Subrosion verstellten Kieskörper ist der Verwitterungsgrad ihrer Kiese. Hierbei zeigen die Grauwacken-Gerölle die weitaus stärkste Verwitterungsanfälligkeit, wobei sich ihre Farbe von grau über oliv nach gelb ändert. Bei den großen Flüssen Sieber und Oder variiert die petrographische Zusammensetzung verschieden alter Kieskörper nur geringfügig und ist deshalb wenig für stratigraphische $Z$ wecke brauchbar. Jedoch zeigen die Nebenbäche, die bisweilen eine beträchtliche laterale Änderung der Kieszusammensetzung innerhalb einer Terrasse hervorrufen können, mit zunehmendem Alter ihrer Kiese eine verstärkte Führung von Rhyolith, besonders im Niveau der Oberterrasse.

In Tabelle 1 sind die wichtigsten Kieskörper und Erosionsterrassen des südwestlichen Harzvorlandes und ihre Stellung zu den Löß-Paläoböden angegeben.

2. Pleistozäne B if urkation der Sieber unmittelbar nach dem Verlassen des Harzes: Das A u e-Trockental (Abb. 1) ist die konsequente Fortsetzung der $45^{\circ}$ streichenden Harzstrecke der Sieber. Der Ausstrich des Zechsteins wird auf kürzestem Wege gequert, das ehemalige Flußbett war nur gering von Subrosionsvorgängen beeinflußt worden. Das westlich hiervon liegende $\mathrm{Hördener-Tal}$ ist die subsequente Fortsetzung der Harzstrecke der Sieber und wird von dieser noch heute durchflossen. Das Tal ist zunächst parallel zum Harzrand in den Zechstein eingetieft und verläuft dann ab Hörden bei Eintritt in den Buntsandstein wieder $45^{\circ}$ streichend parallel zum Aue-Tal. Das Hördener Tal unterliegt im Bereich des Zechsteins in hohem Maß der Subrosion. Hördener Tal und Aue-Tal sind von der Sieber periodenweise gleichzeitig, also bifurkierend durchflossen worden. In Zeiten mit verstärkter Subrosion und geringer fluviatiler Sedimentation wurde aber, wie heute, nur der Hördener Lauf von der Sieber benutzt.

3. Subrosionssenken im Zechstein-Gips, die mit Kiesen über $60 \mathrm{~m}$ Mächtigkeit gefüllt sind, aber durch unterschiedliche Vorgänge entstanden sind: In der ca. $3 \mathrm{~km}$ langen Subrosionssenke westlich von Herzberg (Werra-Anhydrit, Zechstein 1) erfolgte bzw. erfolgt die subrosive Absenkung überwiegend synchron mit der fluviatilen Erosion. Zur Bildung eines mächtigen Kieslagers kam es erst im nachfolgenden Glazial, als die subrosiv-erosiv entstandene Talrinne aufgeschottert wurde. In Gegensatz hierzu verlief die subrosive Absenkung im Pöhlder Becken (Hauptanhydrit, Zechstein 3) überwiegend synchron mit der fluviatilen Akkumulation.

\section{Kieskörper und Flußgeschichte}

\subsection{Oberterrasse}

Verschiedene Beobachtungen sprechen für eine periglaziale Kies-Akkumulation aus der E 1 st e r-Zeit oder einer älteren Kaltphase: Die Oberterrasse ist mit Lößderivaten bedeckt, die nach heutiger Kenntnis wahrscheinlich mit Böden der älteren Saalezeit beginnen (Ricken, im Druck). Ferner liegt die Oberterrasse rd. $50 \mathrm{~m}$ tiefer als die Mittlere Randterrasse des Harzes, die eine kiesfreie Verebnungsfläche darstellt und aufgrund der Funde von Ursus deningeri u. a. in der Einhornhöhle mit mindestens Cromer datiert werden kann (Hövermann 1949; Schütr 1968; Duphorn 1969; Jordan 1976). Die Mächtigkeit des Kieskörpers der Oberterrasse läßt sich wegen weitgehender Erosion nur unvollkommen bestimmen. Ein Mindestbetrag von $17 \mathrm{~m}$ ergibt sich für Oberterrassen-Kiese im Ausstrichbereich des Werra-Anhydrits (Eisenbahnbrücke Kleine Steinau), während die 
Kiese im Bereich des Staßfurt-Dolomits ca. $10 \mathrm{~m}$ betragen (Schloß Herzberg). Eine zweite, subrosiv bedingte Steigerung der Mächtigkeit liegt weiter südlich im jüngeren Hauptanhydrit vor, wo zwischen Hördener- und Aue-Tal wieder mehr als $17 \mathrm{~m}$ Kies erreicht werden. In den südlich gelegenen Buntsandstein-Gebieten treten nicht mehr als $5 \mathrm{~m}$ Kies auf.

Geröllzählungen (Vladi 1976; Ricken 1973, 1980) ergaben drei Provinzen mit unterschiedlicher Zusammensetzung der Kiese (Abb. 1): 1.) Sieber- und Lonau-Kiese süd- und nordwestlich von Herzberg und im Hördener-Tal zwischen Hattorf und Elbingerode mit Vormacht von Grauwacken, daneben Quarzit, Kieselschiefer, Tonschiefer, Rhyolith, untergeordnet Hornfels, Granit und Diabas. 2.) Quarzit-reiche Kiese im NW der Subrosionssenke des Werra-Anhydrits, verursacht durch die Elster-zeitlichen Vorläufer der Steinau, deren heutiges Einzugsgebiet die Ostflanke des Acker-Quarzitzuges abdeckt. 3.) Kiese reich an Rhyolith und Kieselschiefer im Ostteil der Subrosionssenke bei Herzberg, die auf Vorläufer der Sieber-Nebenbäche im Bereich des heutigen Eichelbaches und Eichelngrabens zurückgeführt werden müssen. Die erste und besonders die letzte Geröllprovinz haben gegenüber heute stark erhöhte Rhyolith-Gehalte. VLADI (1976, Abb.4) erklärt dies mit einer damals noch größeren Verbreitung der permischen Rhyolith-Decken im Harz.

Im Aue-Tal sind keine Kiese der Oberterrasse vorhanden. Ebenso fehlen sie an der Nordostflanke des Odertales, so daß nicht eindeutig bewiesen werden kann, ob das AueTal z. Z. der Oberterrasse schon bestanden hat. Allerdings deutet der an Rhyolith reiche Kiesfächer der Geröllprovinz 3 mit seiner Erstreckung zum Odertal an, daß auch schon damals eine östliche Verbindung zur Oder bestanden haben muß. Sieber-Kiese (Quarzit, Granit, Hornfels), die gelegentlich in Provinz 3 auftreten, zeigen außerdem, daß die Sieber mit diesem Kiesfächer randlich in Verbindung stand.

\section{2. Ältere Mittelterrasse}

Die Bezeichnung „Altere Mittelterrasse“ wurde von Vladi (1976) für Sieber-LonauKiese am nordöstlichen Hang der Subrosionssenke bei Herzberg gebraucht, die dort $25 \mathrm{~m}$ bis $30 \mathrm{~m}$ unter dem Niveau der Oberterrasse und $15 \mathrm{~m}$ über den Kiesen der Mittelterrasse liegen. Die Terrassen-stratigraphische Einordnung der Kiese muß vorläufig noch unsicher bleiben, da die Terrassenreste subrosiv verstellt und tiefergelegt worden sind, wie ein neuerer Kanalisationsaufschluß gezeigt hat. Eindeutige Terrassenreste befinden sich aber im Buntsandstein-Gebiet ca. $20 \mathrm{~m}$ unter dem Niveau der Oberterrasse und ca. $10 \mathrm{~m}$ über der Mittelterrasse. Die Mächtigkeit der Kiese beträgt $4 \mathrm{~m}$ (Abb. 2, Profil I). Nach ihrer Lößbedeckung sind die Kiese mindestens in die ältere $\mathrm{S}$ a a le ze it zu stellen und sind wahrscheinlich vor dem Drenthe/Warthe-Interstadial abgelagert worden (Tab. 1). Die Kiese liegen an der Nordostflanke des Odertales und müssen aufgrund ihres Quarzitgehaltes von der Sieber her stammen. Da die Oder nach W entwässerte, können die Kiese nur von der Aue-Sieber geliefert worden sein. Demnach kam es während der Akkumulationsperiode der Alteren Mittelterrasse wahrscheinlich zu einer Bifurkation, oder die Sieber entwässerte nur durch das Auetal.

\subsection{Mittelterrasse}

Etwa $10 \mathrm{~m}$ unterhalb der Alteren Mittelterrasse liegt der Kieskörper der Mittelterrasse, der im Flußgebiet der Sieber zwischen ca. 30 und ca. $8 \mathrm{~m}$ Mächtigkeit erreicht. Der Aufbau seiner Lößdecke zeigt, daß die Kies-Akkumulation der Mittelterrasse m i t o d e r noch vor dem Ende der Warthe-zeitlichen Lößsedimentation beendet war. Die Lößfolgen im Hangenden der Kiese der Mittelterrasse beginnen über- 

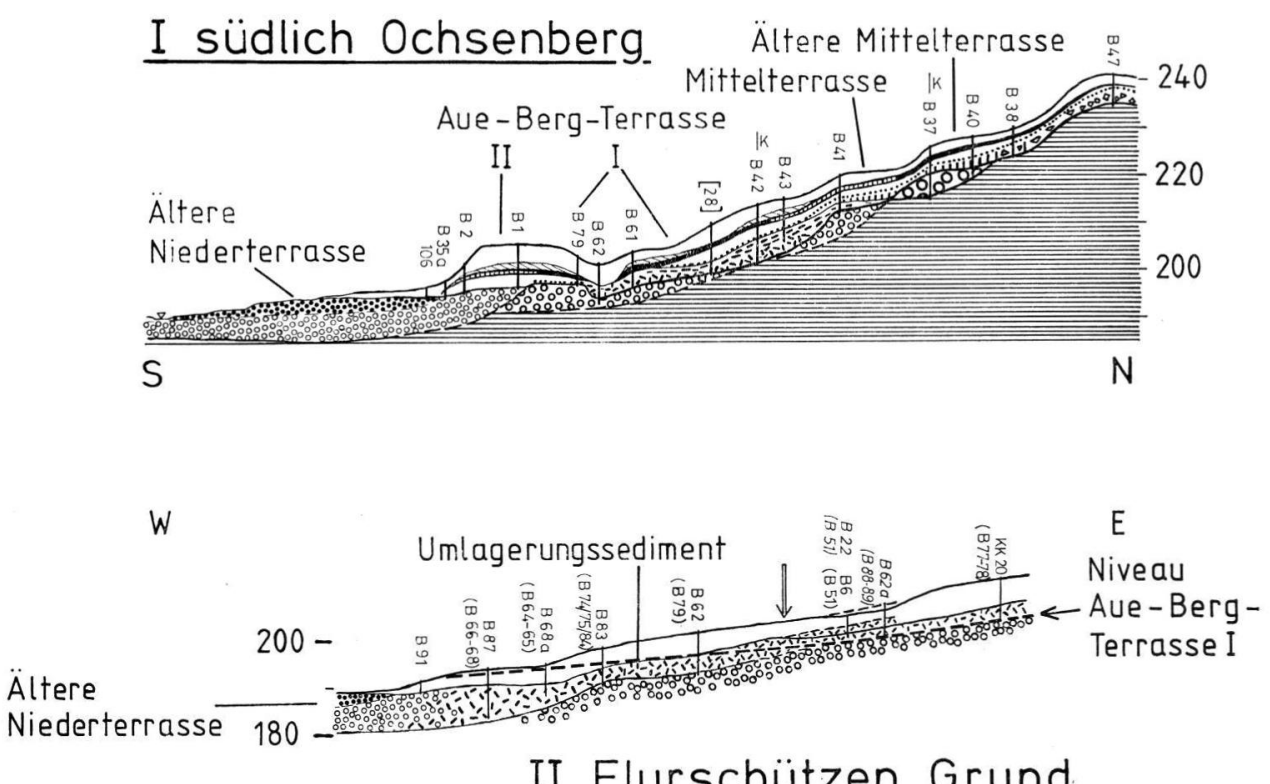

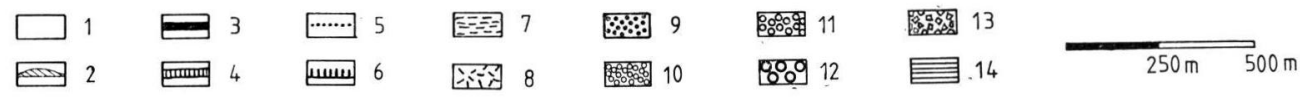

Abb. 2: I Querprofil durch die Kiesterrassen von Oder und Aue-Sieber südlich des Ochsen-Berges. II Längsprofil durch den Flurschützen Grund, Verzahnung von Altweichsel-zeitlichem Umlagerungssediment mit den Kiesen der Alteren Niederterrasse. Pfeil: Verzweigung des Flurschützen Grundes.

Lage der Profile s. Abb. 1. B 42, KK 20: Löß-Bohrung. (B 79): benachbarte Bohrung auf der Aue-Berg-Terrasse I. [28]: Bohrung projeziert. Profile der Löß-Bohrungen in RICKEN (1980) und Ricken (im Druck). - Legende: 1 Lößlehm, entkalkt, Jung- und Mittelweichsel. 2 Löß, Carbonat-haltig, Jungweichsel. 3 Lohner Boden, Mittelweichsel. 4 Hattorfer Boden, Mittelweichsel. 5 oberer Bleichhorizont, Altweichsel. 6 Parabraunerden, Eem bzw. Altweichsel. 7 schluffiges Umlagerungssediment, Altweichsel. 8 Umlagerungssediment mit Harzkiesen, Altweichsel. 9 oberer Kieskörper der Älteren Niederterrasse. 10 unterer Kieskörper der Älteren Niederterasse und Kiese der Aue-Berg-Terrasse II. 11 Kiese der Mittelterrasse. 12 Kiese der Alteren Mittelterrasse. 13 Buntsandstein-Solifluktionsschutt, Altweichsel. 14 Ton-Sandsteine des Unteren Buntsandsteins.

wiegend mit Parabraunerden der Altweichsel-Zeit. Zur Bildung von Eem-Parabraunerden ist es offenbtr nur dann gekommen, wenn im Eem Schwemmlöß seitlich auf die Mittelterrasse transportiert worden ist (Ricken, im Druck). Die Zusammensetzung der Kiese und die große Verbreitung ihrer Sedimente in Hördener- und AueTal belegen eine Bifurkation der Sieber während der Akkumulationsperiode der Mittelterrasse (Abb. 1).

Der einzige bedeutsame Aufschluß in der Schichtenfolge der Mittelterrasse liegt am Prallhang der Sieber $\mathrm{z}$ wischen Hattorf und Elbingerode (Landschaftsschutzgebiet!). Die Schichtenfolge beginnt mit Ton- und Sandsteinen der Unteren Wechselfolge des Unteren Buntsandsteins, die von $5 \mathrm{~m}$ Kies überlagert werden. Darüber folgt $4 \mathrm{~m}$ bis $5 \mathrm{~m}$ Löß (Abb. 3). Die Lößwand enthält fossile Böden, so den obersten 


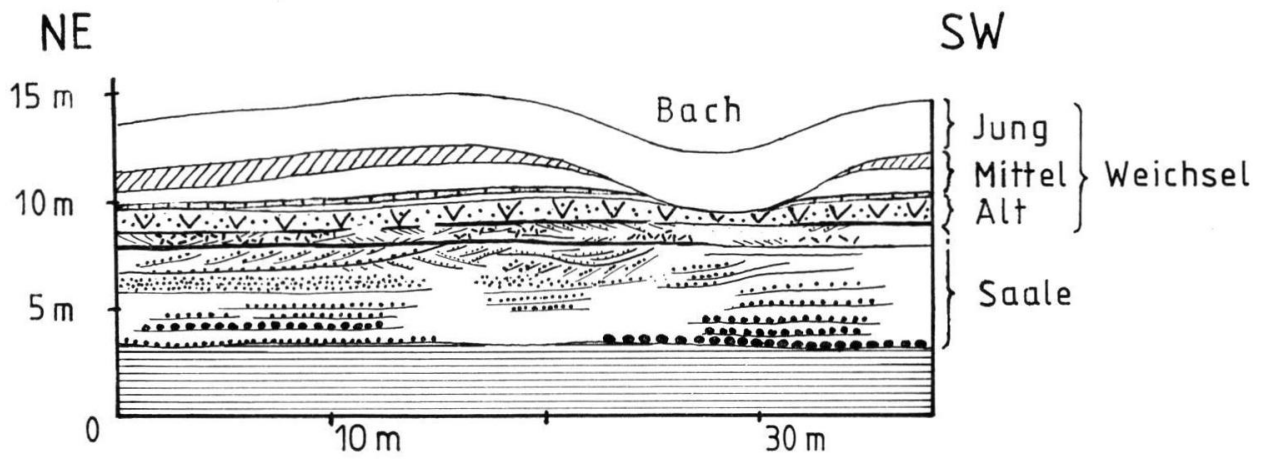

\begin{tabular}{|c|c|c|c|}
\hline & Löß und Lößlehm & $=$ & Schlufflagen \\
\hline सामात्र & $\begin{array}{l}\text { Lohner und Hattorfer Boden } \\
\text { (Stillfried B-Interstadial) }\end{array}$ & ハッ⿻ & $\begin{array}{l}\text { Kiese solifluidal } \\
\text { verlagert }\end{array}$ \\
\hline קחב & Herzberger Boden & 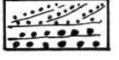 & Schotter, Kiese \\
\hline V:V:V & $\begin{array}{l}\text { oberster Bleichhorizont des } \\
\text { Altweichsels mit Kiesen }\end{array}$ & & $\begin{array}{l}\text { Rotweiße Wechsel- } \\
\text { folge des Unteren }\end{array}$ \\
\hline
\end{tabular}

Abb. 3: Saale- und Weichsel-zeitliche Sedimente am Prallhang der Sieber zwischen Hattorf und Elbingerode, Ausschnitt.

Bleichhorizont des Altweichsels, den Herzberger Boden und, als ein Äquivalent des Stillfried B-Interstadials, den Lohner Boden (Trockenfazies) und Hattorfer Boden (Naßfazies), letzterer ist besonders deutlich ausgebildet $100 \mathrm{~m}$ südwestlich des in Abb. 3 gezeigten Ausschnitts. Für Herzberger und Hattorfer Boden ist die Lößwand zugleich die Typlokalität (RICKEN, im Druck).

Komplexe Parabraunerden des Altweichsel, die direkt im Hangenden der Kiese zu erwarten wären, fehlen. Böden und Kiese sind gegen Ende des Altweichsels durch Solifluktion in einem $1 \mathrm{~km}$ langen und $500 \mathrm{~m}$ breiten Dellental um etwa $3 \mathrm{~m}$ bis $5 \mathrm{~m}$ erniedrigt worden. Daß die Kiese aber ursprünglich von Parabraunerden bedeckt waren, wird durch ihre Umhüllung mit braunem Ton in Form von regelungsdoppelbrechenden Cutanen angezeigt. Der Ton stammt aus der pedogenen Toninfiltration und besteht aus lößbürtigem Smectit und Vermiculit. Die Tonhüllen sind als Folge von Grundwasserhochständen durch Hämatit-Abscheidungen inkrustiert worden, was vermutlich gegen Ende der Saale-Zeit und während der jüngeren Weichsel-Zeit erfolgte. Wegen der Inkrustierung läßt sich der Ton nicht ohne eine vorherige Reduzierung und Lösung des Eisens gewinnen. Die durch die Umkrustung hervorgerufene $\mathrm{r} \ddot{\mathrm{t}} \mathrm{lich}-\mathrm{braune}$ bis rote $\mathrm{Patina}$ der Kiese ist an vielen Stellen typisch für die Ablagerungen der Mittelterrasse.

Obwohl der Kieskörper der Mittelterrasse wegen der solifluidalen Abtragung unvollständig ist, kann an mehreren Stellen des Prallhangs ein - generell gesehen - e i n phasiger und gradierter $\mathrm{Aufbau}$ beobachtet werden, dagegen keine Mehrphasigkeit im Sinne LüTTIGs (1960): 
1. Die Korngröße nimmt von unten nach oben ab. Gerölle und Grobkies gehen in Feinkies und untergeordnet in Sand über. Lößmaterial in Form lang durchhaltender Schluffbänder ("Hochflutlehme") und partiell Fließerden sind nach oben hin eingeschaltet.

2. Die Schichtung verändert sich von ebenen Schichten mit Grobgeröllagen an der Basis zu Schichten mit großdimensionaler Schrägschichtung zu kleindimensional schräggeschichteten Kiesen und Sanden (Abb. 3). Nach Simons et al. (1966) bedeutet dies einen Übergang vom oberen zum unteren Strömungsregime, als Folge abnehmender Fließgeschwindigkeit.

3. Von unten nach oben nehmen die Gehalte an Tonschiefern um ca. $5 \%$ zu, während Grauwacken um ca. 3\% abnehmen, bezogen auf den Anteil von Harzkomponenten in der Fraktion 6,3 mm bis $12,5 \mathrm{~mm}$ (Grundmenge 350 Gerölle). Hierin kommt eine während der Sedimentation zunehmende Aufarbeitung der Kiese zum Ausdruck, die zum Zerbrechen größerer Tonschiefer-Gerölle und wohl auch zu einer selektiven $\mathrm{Ab}-$ fuhr von Grauwacke-Geröllen geführt hat. Diese lassen sich wegen ihrer rundlichen Form und einer durchschnittlichen Dichte von $2,66 \mathrm{~g} / \mathrm{cm}^{3}$ leichter als die plattigen, relativ dichten Tonschiefer $\left(\varrho=2,72 \mathrm{~g} / \mathrm{cm}^{3}\right)$ transportieren.

Insgesamt ist in der Abfolge ein Trend $\mathrm{zu}$ geringeren Abflüssen während der Akkumulation zu erkennen. Hierfür kann ein Kälter- oder Ariderwerden des Klimas die Ursache sein, worauf auch die Fließerdeeinschaltungen im oberen Abschnitt der Kiesfolge hindeuten.

\subsection{Aue-Berg-Terrasse I}

Das Niveau der Aue-Berg-Terrasse I liegt nur wenig höher als die Flächen der jüngeren Aue-Berg-Terrasse II und der Alteren Niederterrasse. Die Aue-Berg-Terrasse I ist durch Erosion mit einem Betrag von $10 \mathrm{~m}$ aus den Kiesen der Mittelterrasse herausgearbeitet worden, ohne daß dabei ein von den Mittelterrasse-Kiesen unterscheidbarer eigener Kieskörper abgelagert wurde. Wegen der deutlichen Erosionsstufe (Abb. 2, Profil I), des flachen Bodens und der girlandenartigen Verbreitung dieser Terrasse auf $4 \mathrm{~km}$ Länge entlang der NE-Flanke des Odertales zwischen Aue-Tal und Hördener Tal (Abb. 1) kann eine fluviatile Anlage durch die Aue-Sieber und Oder nicht ausgeschlossen werden. Allerdings läßt sich das Alter der primären Erosionsterrasse mit Hilfe der Lößstratigraphie nur recht grob zwischen der ausgehenden Saale-Zeit und dem Beginn der Altweichsel-Zeit einengen. Die Oberfläche der Terrasse wird nämlich von U m lag e r u n g s s e d i m e n t e n überprägt, die von zahlreichen Hangtälchen und Dellen ausgehen. Das Umlagerungssediment ist nach Löß-stratigraphischen Befunden in der Altweichsel-Zeit entstanden und besteht zum größten Teil aus Buntsandstein-bürtigem Solifluktionsmaterial und resedimentierten Terrassen-Kiesen. Es wurde in Form von Schwemmfächern auf der Terrasse abgelagert und geht nach $\mathrm{S}$ in eine kleine $\mathrm{T}$ a $1 \mathrm{r}$ in $\mathrm{n}$ e über, die in die Oder entwässert hat (Flurschützen Grund). Dadurch verzahnt sich das Altweichsel-zeitliche Umlagerungssediment des Schwemmfächers mit den $\mathrm{Harz}-\mathrm{Ki}$ esen der Niederterrasse der Oder (Abb. 2, Profil II). Dies wird im folgenden zur chronologischen Eingrenzung der Kiessedimentation der Niederterrasse benutzt.

Wie 34 Bohrungen (Kernbohrungen und Sondierungen) auf der Aue-Berg-Terrasse I zeigen, enthält der obere, zum Hang der Mittelterrasse hin liegende Teil der Schwemmsedimente außerhalb der Talrinnen verlagerte und in situ gebildete Parabraunerde- $\mathrm{B}_{\mathrm{t}}$ Horizonte der Altweichsel-Zeit. In den distalen Bereichen geht die Bildung der Schwemmfächer noch bis in das obere Altweichsel hinein weiter, erkennbar an dem solifluidal ver- 
lagerten und mit Material aus Parabraunerden durchsetzten oberen Bleichhorizont des Altweichsels (Ricken, im Druck, Abb. 12). Dieser Horizont bedeckt auch die Füllung der Dellen und Talrinnen im Bereich ihrer Oberläufe. Jüngere Schwemmbildungen sind bis auf verlagerten Jungweichsel-Löß nicht vorhanden. Der Jungweichsel-Löß durchschneidet in den Hangtälchen den Stillfried B-Horizont (vgl. auch Abb. 3) und liegt im Oberlauf der Talrinne „Flurschützen Grund“ auf Mittel- und Altweichsel-Lößböden. Im Unterlauf hat der Jungweichsel-Löß den älteren Löß völlig durchschnitten und bedeckt direkt das Altweichsel-zeitliche Schwemmfächer-Material. Aus der Verzahnung von Niederterrasse, Tälchenfüllung und Schwemmfächer-Bildungen kann nun folgendes abgeleitet werden:

1. Ein Teil der Alteren Niederterrasse wurde im Altweichsel sedimentiert, Kiese erreichten etwa das Niveau der heutigen Fläche der Niederterrasse.

2. Nach der Bodenbildung des Stillf ried B-Interstadials (ca. 30000 Jahre $\mathrm{BP}$ ) wurde in den Hangtälchen und vermutlich auch im Kieskörper der Niederterrasse erodiert und danach wieder sedimentiert.

Der Zeitpunkt dieser jungen Erosionsphase läßt sich im Untersuchungsgebiet nicht genau festlegen, weil hier die Jungweichsel-Böden nur sehr unvollkommen ausgebildet sind (Tab. 1). Offenbar war die Erosionsphase nur von kurzer Dauer und lag im frühen Jungweichsel, da die im Normalprofil übliche Mächtigkeit der Jungweichsel-Lösse von 3 bis $6 \mathrm{~m}$ ebenfalls in der oben beschriebenen Talrinne erreicht wird, die Hauptmasse der Lößsedimentation jedoch erst im mittleren und späten Jungweichsel erfolgte. Die Erosionsphase dürfte etwa mit der Bildung des ersten Jungweichsel-Bodens zusammenfallen (wj 1Komplex nach Rohdendurg \& Meyer 1966, oder E 1 nach Schönhals et al. 1964). In Mitteleuropa stellt sie eine bedeutende Erosionsperiode in den Hangtälchen dar (SEMMEL 1968: 41 f, Semmel \& Stäblein 1971, Beobachtungen von B. Meyer im Raum Göttingen, freundl. mündl. Mitt.). Nach lößstratigraphischen Befunden kann die Erosionsphase in das Zeitintervall zwischen 30000 Jahren BP (Stillfried B) und 20000 Jahren BP (E 2) eingeengt werden (vgl. ${ }^{14} \mathrm{C}$-Daten bei FinK 1962 und Semmel 1968).

\subsection{Weichsel-zeitliche Schotterkörper und Erosionsterrassen}

\subsubsection{A u e-Berg-Terrasse II}

Die Aue-Berg-Terrasse II liegt als ein maximal $300 \mathrm{~m}$ breiter und $2 \mathrm{~km}$ langer Streifen an der NW-Flanke des Auetales im Mündungsbereich in das Odertal (Abb. 1). Sie ist mit Lößderivaten von über $10 \mathrm{~m}$ Mächtigkeit bedeckt. Ihre Kies-Fläche liegt einen halben Meter unterhalb der Aue-Berg-Terrasse I und etwa einen Meter über der Niederterrasse. Dennoch zeigen die oliv-grauen Verwitterungsfarben der Quarzit-haltigen GrauwackenKiese (5 Y 6/1 bis 6/2 bis 6/3) und ihre fehlende Umkrustung mit Ton und Eisenoxid, $\mathrm{daß}$ es sich hierbei um eine echte Akkumulation der Aue-Sieber von mindestens $2 \mathrm{~m}$ Mächtigkeit handelt und nicht um die Relikte des Kieskörpers der Mittelterrasse (Abb. 2, Profil I).

Die Lößfolge, die mit dem Eijkelkamp-Kombinationsbohrer untersucht wurde, beginnt auf der Aue-Berg-Terrasse II mit dem Mittelweichsel, noch vor Bildung des Herzberger Bodens (Ricken, im Druck). Die obersten dm der Kiese enthalten stellenweise Bleichhorizont-Material und die ebenfalls für das Altweichsel typischen Holzkohlen von Nadelhölzern, darunter Pinus sp.. Demnach handelt es sich bei dem Kieskörper der Aue-BergTerrasse II um eine Akkumulation der frühen Weichsel-Zeit. Wie weiter unten noch begründet werden soll, dürfte die Aue-Berg-Terrasse II einen von späterer Erosion verschont gebliebenen Rest des unteren Kieskörpers der Alteren Niederterrasse darstellen. 
Der ca. $2 \mathrm{~m}$ über den Kiesen im Löß liegende Bodenhorizont des Stillfried B-Interstadials ist nicht in seiner Trockenfazies (Lohner Boden), sondern als $\mathrm{Na}$ ß fazies (Hattorfer Boden) mit frostplattigem Untergrund ausgebildet.

\subsubsection{Steie-Terrasse}

Die Steie-Terrasse ist eine kleinere jüngere Erosionsstufe am Ende des Mittelweichsels in den Kiesen der Mittelterrasse. Hier liegen im südöstlichen Mündungsbereich des Auetales in die Oder (Abb. 1) bis zu $3 \mathrm{~m}$ mächtige allochthone und autochthone Pakete des Hattorfer Bodens, etwa niveaugleich mit der Oberfläche der Älteren Niederterrasse. Die Vernässung auf den flußnahen Terrassenflächen zeigt an, daß das Grundwasser nach dem Trockenfallen der Aue-Berg-Terrasse II während des Stillfried B-Interstadials nicht wesentlich abgesunken war und kapillarer Wasseraufstieg aus den Kiesen in den Löß möglich war. Das Flußbett der Sieber war also während des Stillfried B-Interstadials nur unbedeutend unter das Niveau der AueBerg-Terrasse I I eingetief t worden (vgl. auch Abb. 6).

\subsection{3. $\ddot{\mathrm{A}} 1 \mathrm{t}$ ere $\mathrm{Niederterrasse}$}

Die großflächige Verbreitung der Kiese der Alteren Niederterrasse im Hördener-Tal und im Aue-Tal (Abb.1, Abb. 4), sowie ihre petrographische Zusammensetzung zeugen für eine Weichsel-zeitliche Bifurkation der Sieber. Wie in zahlreichen Kiesgruben zu sehen ist, besteht die Ältere Niederterrasse aus zwei sich diskordant überlagernden Sedimentkörpern (Tafel 1, Fig. 1, 2 und 3). Der untere Sedimentkörper kann, je nach seiner Lage zu Subrosionsgebieten, in seiner Mächtigkeit von ca. $1 \mathrm{~m}$ (westlich Hattorf) bis zu mehreren Zehnern von Metern (Pöhlde) schwanken, während der obere Kieskörper eine etwa konstante Mächtigkeit von 2,5 $\mathrm{m}$ bis $4 \mathrm{~m}$ aufweist.

Un t e r e r K i es k ör per (Abb. 5): Die Verwitterungsfarben der Grauwacken entsprechen denen der Aue-Berg-Terrasse II. Weitdurchhaltende Schichten und Erosionsrinnen, die mit Stillwasserschluffen gefüllt sind, sind typisch. Den Kiesen ist dunkelbraunes To n material beigemengt, welches offenbar aus erodierten Parabraunerden stammt und das gesamte Kiespaket rötlich-braun färbt. Die durchschnittliche Korngrößenverteilung in den Kiesen ändert sich nicht trendhaft, jedoch war in der ehemaligen Kiesgrube

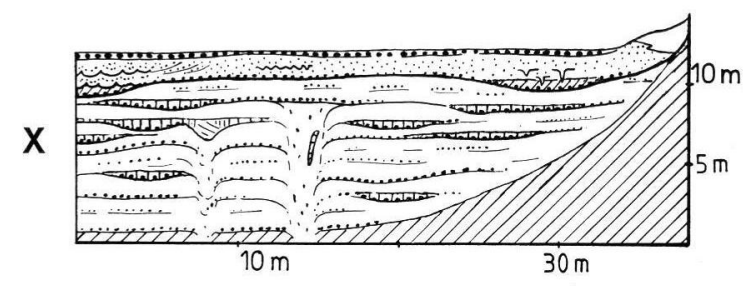

Kryoturbation, Eiskeilpseudomorphose
Lößfall, syngenetisch
oberer Jungweichsels (lokal im Hangbereich)
Unterer Kieskörper

Abb. 5: Aufbau der Älteren Niederterrasse zwischen Herzberg und Hörden, schematisiert.

$X$ Fundniveau von Holzkohlen in der ehemaligen Kiesgrube Hörden. 
bei Hörden nach oben eine zunehmende Sedimentaufarbeitung festzustellen (Zunahme von Tonschiefern, Abnahme von Grauwacken). S y n gen e t is che Erdfalls ch lote mit ihren Füllungen zeigen in der ehemaligen Kiesgrube bei Hörden, daß die s u b r osive Absenkung in dieser Periode noch nicht zur Ruhe gekommen war.

Gelegentlich enthalten Stillwasser-Sedimente in den Kiesen bis 0,5 cm große Stücke von Holzkohlen. Eine Bestimmung durch Herrn Prof. Willerding (Göttingen) ergab: Ulmus (8), Juniperus (5), Betula (2) (Kiesgrube $500 \mathrm{~m}$ westlich Pöhlde); Larix (7), Pinus (3), Nadelbolz (1) (ehem. Kiesgrube Hörden). Alle Holzkohlen haben außerordentlich kleine Jahresringe, die schlechte Wuchsbedingungen anzeigen. Die Assoziation von Ulmus mit Juniperus und Betula ist nur schwer interpretierbar. Wenn es sich nicht um teilweise umgelagerte Eem-Kohlen handelt, muß Ulmus in das Frühweichsel bzw. A $1 \mathrm{t}$ w e i chs el der Lößstratigraphie gestellt werden. Ähnlich verhält es sich mit Larix, von der kleine Zwergformen bekannt sind. Für eine Einordnung in das Frühweichsel sprechen die bisher erarbeitete Vegetationsgeschichte in Norddeutschland und den Niederlanden (z. B. Wijmstra 1978; Mania \& Töpfer 1973; Behre 1974), aber auch eigene Daten. In einem Erdfall $3 \mathrm{~km}$ westlich von Herzberg liegt eine Schichtenfolge, die vom Eem-Interglazial über ein Frühweichsel-Interstadial noch weiter in die Weichsel-Zeit hineinreicht und vom Hattorfer Boden überlagert wird. Erste Ergebnisse der pollenanalytischen Bearbeitung durch Dr. Grüger (Palynologie Göttingen) weisen für das Frühweichsel folgende Baumarten nach: Juniperus, Betula, Pinus, Picea und Larix. Eine jüngere Einordnung der Holzkohlen als Frühweichsel ist nicht möglich, da am Südharz Mittel- und Jungweichsel weitgehend baumfrei waren. Das wird daraus ersichtlich, daß während des Stillfried B-Interstadials, in dem es zur markantesten Bodenbildung innerhalb der Löß-Boden-Folge von Mittel- und Jungweichsel gekommen ist, sich lediglich eine Gras/Kraut-Tundra mit vermutlich Zwergformen von Salix, Betula und Pinus entwickelte (RICKeN, im Druck).

a) Paläobotanische Befunde und b) die in Kapitel 2.4. beschriebene Verzahnung von Altweichsel-zeitlichen Umlagerungssedimenten mit den Kiesen der Älteren Niederterrasse zeigen, daß der untere Ki skörper der Alteren Niederterrasse ein überwiegend Alt weichsel-zeitliches Alter besitzen muß. Die Kiessedimentation der Aue-Berg-Terrasse II erfolgte nach lößstratigraphischen Ergebnissen ebenfalls im Altweichsel. Außerdem besitzen beide Kieskörper denselben Verwitterungsgrad und liegen benachbart auf etwa einem Niveau. Die Kieskörper der Aue-Berg-Terrasse II und der des unteren Teiles der Alteren Niederterrasse müssen deshalb Bildungen mit identischem Schotterkörper sein.

\section{T a f e 11}

Fig. 1: Ehemalige Kiesgrube $1 \mathrm{~km}$ nordöstlich Hörden. Der untere Kieskörper der Älteren Niederterrasse wird vom jüngeren Kieskörper diskordant überlagert (Pfeile).

Fig. 2: Ausschnitt aus A. Erosionsdiskordanz zwischen unterem und oberem Kieskörper der Alteren Niederterrasse. Nach der Erosionsphase werden Stillwasser-Sedimente abgelagert (Pfeil), darauf erfolgt erneute Kiessedimentation.

Fig. 3: Ehemalige Kiesgrube $150 \mathrm{~m}$ südlich vom Auekrug in der Niederterrasse. Ca $2 \mathrm{~m}$ über dem Wasserspiegel wird der untere Kieskörper der Älteren Niederterrasse sichtbar (Pfeile).

Fig. 4: Ehemalige Kiesgrube in der Oderaue $1 \mathrm{~km}$ nordöstlich Pöhlde. Der schräggeschichtete obere Kieskörper der Älteren Niederterrasse wird von Kiesen der Jüngeren Talaue überlagert (untere Pfeile), die nach einer Groblage im oberen Teil Hüttenschlacken enthalten (Obere Pfeile). Weißer Balken jeweils $1 \mathrm{~m}$. 

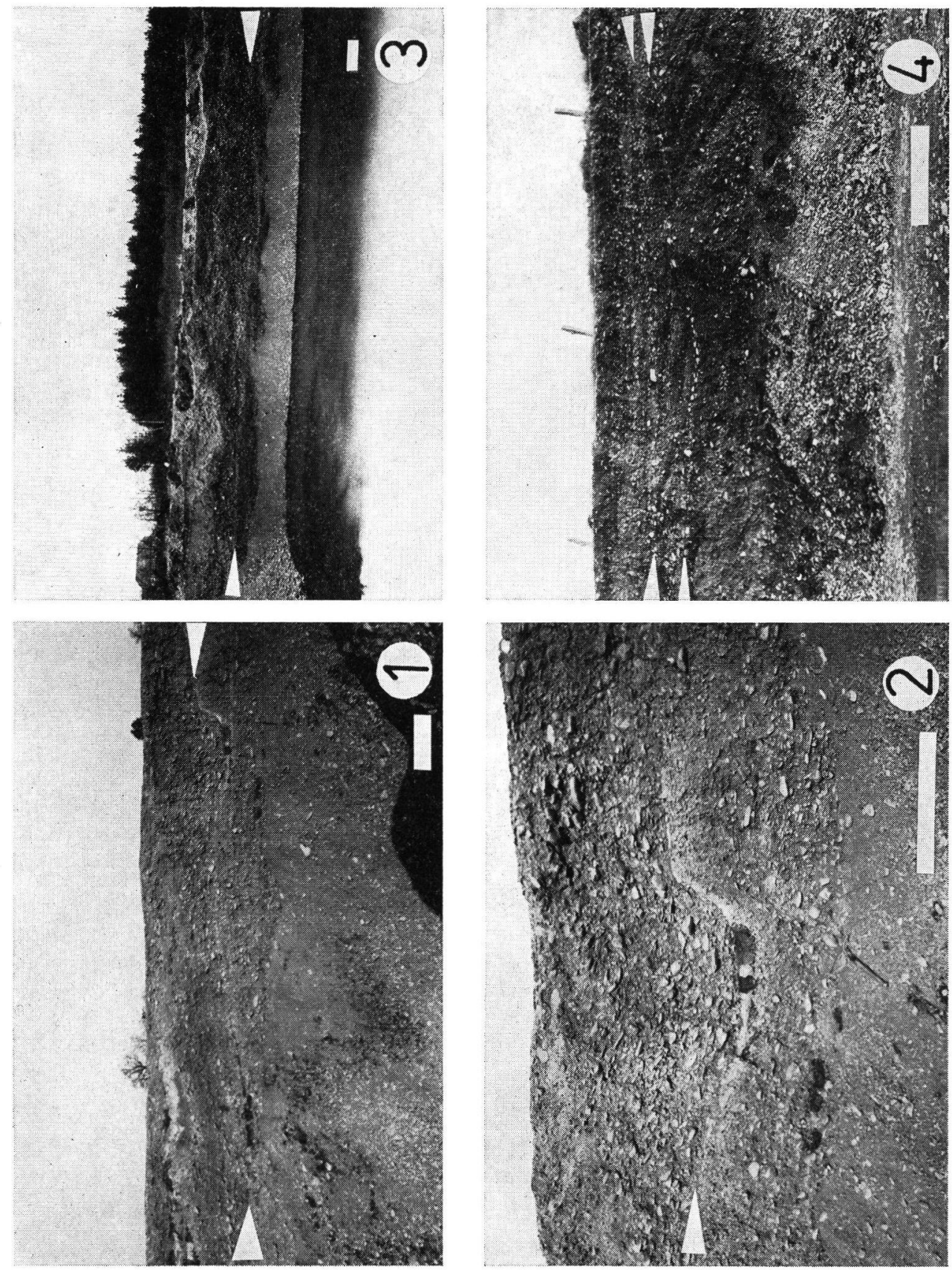
Der Kieskörper der Aue-Berg-Terrasse II ist wahrscheinlich die Fortsetzung des unteren Kieskörpers der Alteren Niederterrasse, dessen Oberfläche in der Aue-Berg-Terrasse II lediglich nicht auf das Niveau des unteren Kieskörpers der Alteren Niederterrasse abgetragen worden ist. Der inaktive, vom Fluß nicht mehr benutzte Teil des gemeinsamen Kieskörpers wurde dann seit Ende des Altweichsels äolisch mit ca. $10 \mathrm{~m}$ Löß bedeckt (Abb. 2, Profil I, vgl. auch Abb. 6).

Oberer Kieskörper (Abb. 5): Sehr langaushaltende ebene Schichtung mit Dachziegellagerung, oben öfter aus gering aufgearbeiteten Lagen mit Grobgeröllen. Der obere Kieskörper beginnt an seiner Basis vielerorts mit Stillwassersedimenten, die Vergleyung zeigen können (Taf. 1, Fig. 2). In Talrand-Lagen verzahnt sich der obere Teil des Kieskörpers stellenweise mit dem JungweichselL öß (Hörden, Prallhang der Sieber, $400 \mathrm{~m}$ südlich der oberen Brücke). Auffälligstes Merkmal des Kieskörpers ist das Fehlen von braunem Lehmmaterial, das ihn farblich vom unteren Kieskörper abhebt. Die Grauwacken sind nur leicht verwittert und haben im trockenen Zustand graue bis dunkelgraue Farben (5 YR 5/1 bis 10 YR 4/2). S y n g e net ische Erdfallbildugen konnten trotz zahlreicher Aufschlüsse $\mathrm{nicht}$ be obachtet werden, dafür aber in mehreren Fällen kleine Eiskeile und Kryo$\mathrm{t} u \mathrm{rb}$ a t i o n e $\mathrm{n}$, besonders in Schlufflagen. Solche Frostanzeiger treten im Jungweichsel verstärkt auf (ROHDENBURG 1966).

Für die Dauer der Sedimentation des oberen Kieskörpers der Alteren Niederterrasse dürfte seiner Verzahnung mit dem Jungweichsel-Löß zufolge fast die gesamte Jungweichsel-Zeit zu veranschlagen sein und zwar vermutlich von der Erosionsphase ab, die nach dem Stillfried B-Interstadial anzusetzen ist. Die Tieferschaltung der Sieber vor der Sedimentation des oberen Kieskörpers erfolgte offenbar in voller Talbreite. Es läßt sich keine Unterstufe der Alteren Niederterrasse der Sieber nachweisen, die mit Jungweichsel-Löß bedeckt ist. Solche Flächen sind aber im Bereich der Söse, des westlichen Nachbarflusses der Sieber, vorhanden. Ein Kilometer nördlich von Eisdorf liegen im Mündungsbereich von Markau und Schlungwasser in das Sösetal Kiesflächen von insgesamt $1 \mathrm{~km}$ Länge und $500 \mathrm{~m}$ Breite, die niveaumäßig etwa in Höhe der Niederterrassen-Fläche liegen bzw. seitlich in diese übergehen und von Lößderivaten bis 3,50 m Stärke bedeckt sind. Jordan (1972) hält diese Flächen wegen ihrer Lößdecke noch für die subrosiv abgesenkte Mittelterrasse. Wie jedoch ein Aufschluß und Bohrungen auf dem südlichen Flächenstück zeigen, gleicht der Verwitterungszustand der Grauwacken mit 5 Y $5 / 1$ bis $5 / 2$ bis $5 / 3$ völlig denen der benachbarten Niederterrasse, nicht dagegen den stärker verwitterten Grauwacken der Mittelterrasse. Die solifluidal geprägte Löß-Folge beginnt hier nicht mit den für die Mittelterrasse typischen Parabraunerden des Altweichsels, sondern gleich mit jüngeren Lössen: Auf den höchsten Teilen des untersuchten südöstlichsten Flächenstücks liegt noch der in situ gebildete und teilweise verlagerte Hattorfer Boden. Dies entspricht der Erosionsphase, die im Tal der Aue-Sieber zur Bildung der Steie-Terrasse führte. Der größte Teil der Fläche wird jedoch von Jungweichsel-Löß eingenommen, in dem verschiedene Naßböden entwickelt sind. Dies entspricht im Siebertal der Abtragung vor der Sedimentation des oberen Kieskörpers während der Erosionsphase am Beginn des Jungweichsels (Abb.6).

Eine $\mathrm{Z}$ weigliedrigkeit des Schotterkörpers der Alteren Niederterrasse ist auch von anderen Flüssen beschrieben worden, teilweise mit ähnlichen Ergebnissen:

1. Erosionsdiskordanz in den obersten Metern der A lteren Niederterrasse: Steinmüller (1965), Zorge bei Nordhausen, Oberkante des unteren Kieskörpers nach ${ }^{14} \mathrm{C}$-Bestimmung 21430 und 22500 Jahre BP. SCHEER (1978), Main, Basis des oberen Kieskörpers nach ${ }^{14} \mathrm{C}$-Bestimmung 15130 und 18300 
Jahre BP. Löscher et al. (1980), Rhein, Oberkante des unteren Kieskörpers nach ${ }^{14} \mathrm{C}$-Bestimmung 27080 Jahre BP.

2. Bildung einer jüngeren Terrassenstufe und Bedeckung der älteren Terrassenstufe mit äolischem Löß: Kulick \& Semmel (1968), Netze in Waldeck, höhere Teile der Älteren Niederterrasse mit $5 \mathrm{~m}$ Löß bedeckt, noch vor dem E 2-Boden beginnend. SEmmel (1972), Werra bei Wölfershausen, höhere Teile der Niederterrasse mit mehreren Metern Löß bedeckt, noch vor dem E 2-Boden beginnend. B. MEYER (freundl. mündl. Mitt.), Leine bei Göttingen, Niederterrasse mit ca. $2 \mathrm{~m}$ Löß bedeckt, an der Basis wj 2 B-Boden im Löß. (Für den E 2Boden, der dem wj $2 \beta$-Boden Niedersachsens entspricht, gibt Semmel (1968) ein ${ }^{14} \mathrm{C}$ Alter von rd. 20000 Jahren BP. an.) Semmel (1974), Seidenschwann (1980), Main, höhere Teile der Alteren Niederterrasse ( $t$ 6) von Löß bedeckt, darin Eltviller Tuff. BRUnotTE (1979), Leine bei Einbeck, nach E exponierte Randbereiche der Älteren Niederterrasse von mehreren Metern Jungweichsel-Löß bedeckt.

Im südwestlichen Harzvorland ist die Fläche des oberen Kieskörpers der Älteren Niederterrasse nicht völlig frei von Decksedimenten. Von Auelehmbildungen abgesehen liegen auf der Kiesfläche, besonders in ihren Randbereichen, zu etwa einem Viertel 0,5 m mächtige lößbürtige Sedimente, die auf den jüngeren Terrassen fehlen. Es handelt sich hierbei um lokale Bildungen, die gegen Ende des Jungweichsels entstanden sein müssen, als sich die Flüsse erst mit dem Nachlassen der äolischen Lößakkumulation von den breiten Flächen der Niederterrasse zur ückz o g e n. Im südwestlichen Harzvorland zeigen vor allem die Decksedimente in Harznähe eine solifluidale Prägung (vgl. auch Jordan 1976). Daneben gibt es Bereiche, die äolischem Löß recht nahe kommen, was auch in anderen Gebieten im Mittelgebirgsraum beobachtet wurde (Steinmüller 1965, Zorge; Wildhagen \& Meyer 1972, Leine; Unger 1974, Thüringen).

Reliktlauf im Aue-Ta l: In die unzertalte Fläche der Alteren Niederterrasse des Aue-Trockentales ist ein System von ca. $0,5 \mathrm{~m}$ bis $1 \mathrm{~m}$ tiefen a $\mathrm{n}$ a s to mos i e r e $\mathrm{n}$ den Rinnen eingeschnitten, die kurz vor dem letzten Versiegen des Auelaufes entstanden sind (Abb. 4). Die jüngste Rinne liegt zentral und ist nicht mehr von dem oben beschriebenen Decksediment überlagert. Nach der von RösseRT (1976: 40) erweiterten STRICKLER-Formel läßt sich der Abfluß für ein reliefschaffendes Hochwasser in dieser Rinne mit ca. $50 \mathrm{~m}^{3} / \mathrm{sec}$ bis $60 \mathrm{~m}^{3} / \mathrm{sec}$ schätzen. Der Gesamtabfluß der Sieber (Aue-Tal und Hördener Tal) muß jedoch mindestens das Doppelte betragen haben, da die Sieber zu dieser Zeit noch bifurkierte, also $100 \mathrm{~m}^{3} / \mathrm{sec}$ bis $120 \mathrm{~m}^{3} / \mathrm{sec}$. Das größte rezente Hochwasser in einer 30jährigen Beobachtungszeit hatte einen Abfluß von $70 \mathrm{~m}^{3} / \mathrm{sec}$ (HAASE et al. 1970). Der Hochwasserabfluß im a usgehenden Jungweichsel war demnach größer als der heutige, warmzeitliche.

Es muß vorläufig offen bleiben, warum das Aue-Tal kurz nach dem Ende der äolischen Lößsedimentation an der Wende Jungweichsel/Spätweichsel schon unter kaltzeitlichen Klimaverhältnissen aufgegeben wurde, so daß keine holozäne Einschneidung mehr erfolgte. Diese Beobachtung steht im Gegensatz zu den Befunden in älteren Abschnitten des Pleistozäns, wo die Sieber nach einer Akkumulationsphase jeweils noch über längere Zeit gleichzeitig in Hördener-und AueTal erodierte, ehe das Aue-Tal schließlichzugunsten des Hördener Tales a ufgegeben w urde. Möglicherweise sind hierfür erste Erdfälle im ehemaligen Verzweigungsbereich der Sieber verantwortlich zu machen. Die jüngste Periode der Erdfallbildung beginnt im südlichen Harzvorland unter kaltzeitlichen Bedingungen, wahrscheinlich nach dem Tauen des Dauerfrostes (Ricken 1980: 50), (Abb. 6). 
Die im Spätweichsel und Holozän folgenden Terrassen und Talauen sind nur noch im Hördener-Tal, besonders in der Subrosionssenke bei Herzberg und z. T. auch im Odertal ausgebildet. Sie fehlen im Aue-Tal, weil dieses in dieser Zeit trocken liegt.

\subsubsection{J üngere $\mathrm{N}$ iederterrasse}

Sie ist in der Subrosionssenke bei Herzberg zweiphasig insgesamt ca. $3 \mathrm{~m}$ in die Altere Niederterrasse eingetieft und war im Bereich von Profil C Abb. 4 mit einem $1 \mathrm{~m}$ mächtigen Umlagerungskörper aus Grobgeröllen aufgeschlossen. Die Jüngere Niederterrasse ist deshalb weitgehend als eine Erosionster r a s e anzusprechen, wobei die Lateralerosion durch Aufflaserung der Sieber in kleine, sich verzweigende und wieder vereinende Nebenarme einen großen Anteil gehabt haben dürfte. Die Terrassen-Ränder sind deshalb, ähnlich wie bei der Alteren Niederterrasse, gestreckt. Rinnen, die den Abfluß auf wenige Aktivitätsbereiche beschränkten, hat es nicht gegeben, im Gegenteil: Westlich von Herzberg kommt es im Gebiet des Eichholzes zu einer 1,5 km langen Bifurkation (Abb. 4).

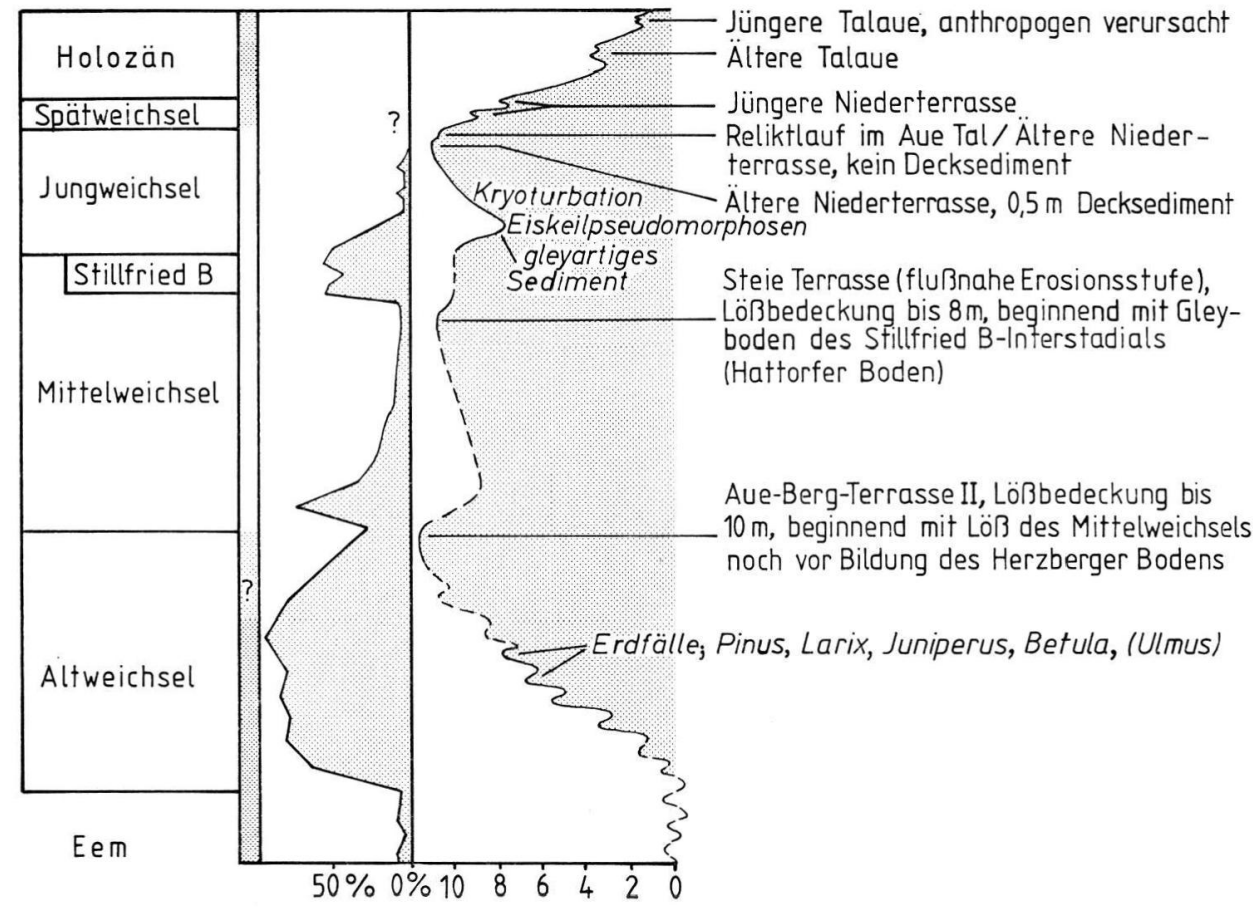

Abb. 6: Sedimentationsdiagramm für jungpleistozäne und holozäne Terrassen-Kiese der Sieber zwischen Herzberg und Hörden. Spalte 1: Löß-Stratigraphie. Spalte 2: Intensität der subrosiven Absenkung, schematisiert. Spalte 3: Intensität der Solifluktion im Hangbereich (Häufigkeit von Sediment-Streifung in Löß-Profilen, nach RICKEN [im Druck]), mit einem Maximum der Solifluktion im Altweichsel (Parabraunerden) und Nebenmaxima zur Zeit des Herzberger Bodens und vor und nach dem Stillfried B - Interstadial (kryoklastische Böden). Spalte 4: Mächtigkeit von Terrassen-Kiesen über der Kies-Basis der Alteren Niederterrasse. Die Kurve gibt lediglich die jeweilige Sediment-Höhe an (in Metern), die etwa der Grundwasser-Oberfläche im flußnahen Bereich entspricht, nicht aber die akkumulierten bzw. erodierten Sediment-Volumina. Der ausgeprägten Aufschotterung im Altweichsel während des Solifluktions-Maximums entspricht die kräftige, durch synerosive Subrosion gesteuerte Eintiefung im Spätweichsel und Holozän. Kleinere Erosionsphasen im Mittel- und Jungweichsel, sowie ein Teil der Erosion im Spätweichsel sind an Tundren-Klima gebunden, teilweise mit kryoklastischer Bodenbildung. 
Die pollenanalytische Datierung der Basis einer Erdfall-Füllung auf der Jüngeren Niederterrasse ergab vorläufig ein früh-holozänes bis spätglaziales Alter. (Weiterführende Untersuchungen an diesem Erdfall werden z. Z. von Frau CHen, Palynologie Göttingen, durchgeführt). Die Entstehungszeit der Jüngeren Niederterrasse kann vorerst zwischen $14000-13000$ Jahren BP (Altere Niederterrasse) und etwa 10000 Jahren BP eingeengt werden. Damit unterscheidet sich die Sieber nicht von anderen Flüssen Mitteleuropas, wo vielerorts eine spätglaziale Erosions- und Aktivitätsphase nachgewiesen worden ist, hauptsächlich durch verlagerten Laacher Bims, wie z. B. im Leinetal (WILdHAGEN \& MeYer 1972).

\subsection{Holozäne Terrassenkiese}

\subsection{1. $\ddot{A} 1$ tere Talaue}

Die Ältere Talaue ist mit mäanderförmig gebogenen Erosionsrändern in die älteren Bildungen eingetieft und erreicht in der Subrosionssenke bei Herzberg Erosionsbeträge gegenüber der Älteren Niederterrasse von 4 bis $10 \mathrm{~m}$. In Profil C der Abb. 4 war in der Älteren Talaue ein $2 \mathrm{~m}$ mächtiger, geröllreicher und mit Lehm durchsetzter Schotterkörper aufgeschlossen. Das Alter liegt zwischen größer 10000 Jahren BP (Jüngere Niederterrasse) und rd. 2000 Jahren BP (Beginn der jüngstholozänen Sedimentation in der Oderaue). Wahrscheinlich ist der größte Teil der Kiese jünger als das untere Atlantikum, da die Talaue bei Pöhlde in eine Erdfallreihe einbiegt, die etwa in dieser Zeit entstanden sein dürfte (RICKEN 1980).

\subsubsection{Jüngere Talaue}

Die Feinkiese der Jüngeren Talaue begleiten eng die heutigen Flußläufe. Sie sind bei Pöhlde zweiphasig aufgeschottert, beginnen jeweils mit einer Groblage und können insgesamt 2,5 m mächtig werden (Taf. 1, Fig. 4). Der untere Teil konnte $1 \mathrm{~km}$ westlich Pöhlde durch eine ${ }^{14} \mathrm{C}$-Bestimmung auf $1780 \pm 55$ Jahre BP datiert werden (Holz, 1,6 m unter Flür, Nieders. L.A. f. Bodenforsch. Labor Nr. Hv 9289). Der obere Feinkies-Körper, enthält wie viele Harzbäche Hüttenschlacken und dürfte der Periode intensiver Eisenverhüttung vom 16. bis zum Beginn des 18. Jahrhunderts entsprechen, als es zu einer weitgehenden Entwaldung in den Tälern von Oder und Sieber kam (Hillegeist 1977).

\subsection{Flußgeschichte}

Bei der Flußgeschichte der Sieber geht es vor allem um das Auffinden der Perioden, in welchen die Sieber bifurkierte bzw. kurzfristig und wiederholt von einem Tal in das andere wechselte und in welchen sie dauerhaft nur einen Flußarm benutzte. Wie oben bereits dargestellt, bifurkierte die Sieber in allen Phasen größerer $\mathrm{Kies}-\mathrm{Akkumulation,} \mathrm{vermutlich} \mathrm{auch} \mathrm{während} \mathrm{der} \mathrm{Bildung} \mathrm{der} \mathrm{Oberterrasse.}$ Verglichen mit den Akkumulationsphasen ist es jedoch schwierig zu ermitteln, ob die Sieber auch in Phasen der Tiefenerosion bifurkierte.

Dazu kann man vereinfacht die Basis eines Kieskörpers als das Niveau auffassen, auf dem der Fluß am Ende seiner Erosionsphase floß, kurz bevor der neue Kieskörper sedimentiert wurde. Läge die Kies-Basis im Aue- und Hördener-Tal auf gleichem Niveau, so wären beide Arme gleichwertig benutzt worden. Lägen die Kiese eines Laufes in der Nähe der Verzweigungsstelle auf tieferem Niveau, so wäre dieser Lauf wegen seines partiell steileren Gefälles bevorzugt worden. Die Oberflächen der Kieskörper können sich dagegen nicht in ihrer Höhenlage unterscheiden, da wegen der Bifurkation am Ende der Akkumulationsphase keiner der beiden Flußläufe ein bevorzugtes Gefälle besaß. 


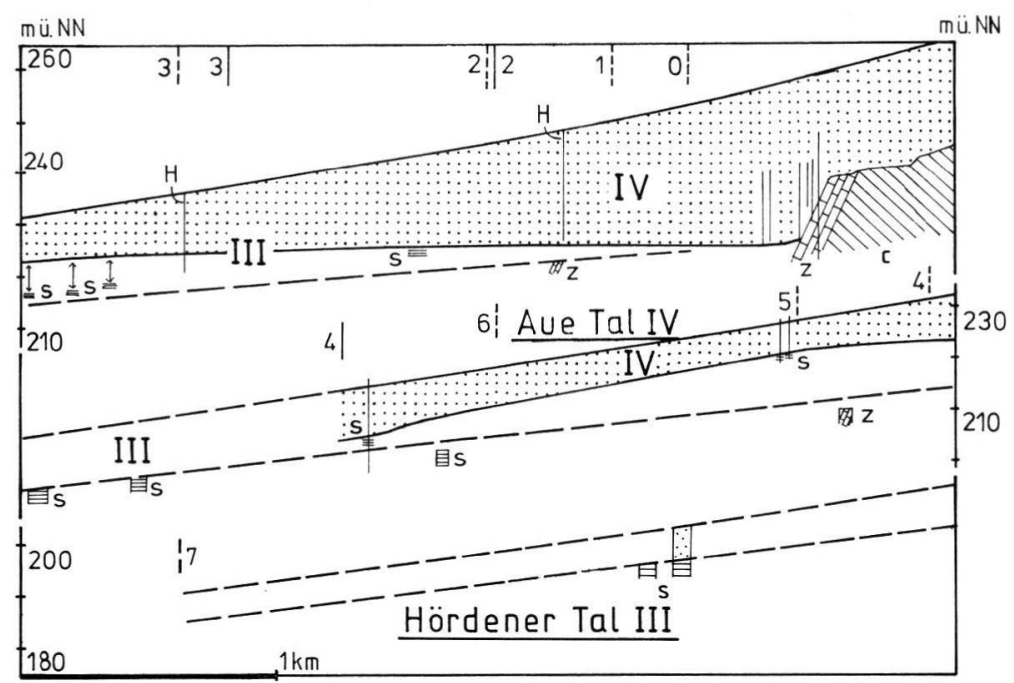

Abb. 7: Kieskörper der Mittelterrasse, Längsprofile für Hördener Tal (III) und Aue Tal (IV). Profilverlauf, Bohrungen und Aufschlüsse s. Abb. 1. Knicke im Profil sind für das Aue Tal mit durchgezogenen Linien, für das Hördener Tal mit gestrichelten Linien angegeben. Bohrungen ergänzt nach Vladi (1976), SEedorf (1955) und Piehler (1969). Die Profile sind übereinander liegend dargestellt, so daß die Oberflächen der beiden Kieskörper zusammenfallen. Aufschlüsse: Kästen. s: unterer Buntsandstein. z: Zechstein. c: Karbon.

Abb. 7 und 8 zeigen Längsprofile durch die beiden Bifurkations-Täler der Sieber. I m Hördener-Tal werden Mittel-und Niederterrasse doppelt so mächtig wie im Aue-Tal, während die Oberflächen der beiden. $\mathrm{Ki}$ eskörper a uf einem Niveau liegen. Aus diesem Befund können aber flußgeschichtliche Aussagen nur dann abgeleitet werden, wenn ...

1. die Längsprofile auch die tiefste Lage der Basis geschichteter Kiese angeben. Im Stadtgebiet von Herzberg, wo Aufschlüsse weitgehend fehlen, kann nicht zwischen geschichteten und verstürzten Kiesen, die in Form von Erdfall-Schlot-Füllungen den Untergrund durchsetzen, unterschieden werden. Über die Kiesmächtigkeiten der Mittelterrasse ist noch zu wenig bekannt, vor allem auf dem Areal zwischen Aue- und Oder-Tal. Für die Niederterrasse ist aber der verdickte Kieskörper im Hördener-Tal und im Aue-Tal durch vollständige Querprofile belegt (Kiesgruben, Hammerschlagseismik, Bohrungen; Abb. 4).

2. der Fluß am Ende einer Erosionsphase wirklich im Niveau der Basis des darauf akkumulierten Kieskörpers floß und nicht auf einem höheren Niveau. Seine Kiese könnten in einem späteren Stadium der Akkumulation großflächig subrosiv, d. h. ohne die Schichtung zu zerstören, abgesenkt worden sein. Solchen Vorgängen scheint aber im Siebertal keine vorrangige Bedeutung zuzukommen, da sich die ges chich te te n Kiese der Alteren Niederterrasse des Hördener Tales in großer Mächtigkeit bis in das Harz-Paläozoikum hinein fortsetzen. An der Mündung der Lonau liegen mindestens $18 \mathrm{~m}$ geschichtete Kiese der Lonau-Niederterrasse auf Zechsteinkalk und Grauwacken in einer verschütteten Schlucht. Diese Kiese können nicht subrosiv abgesenkt worden sein. S i e be r 

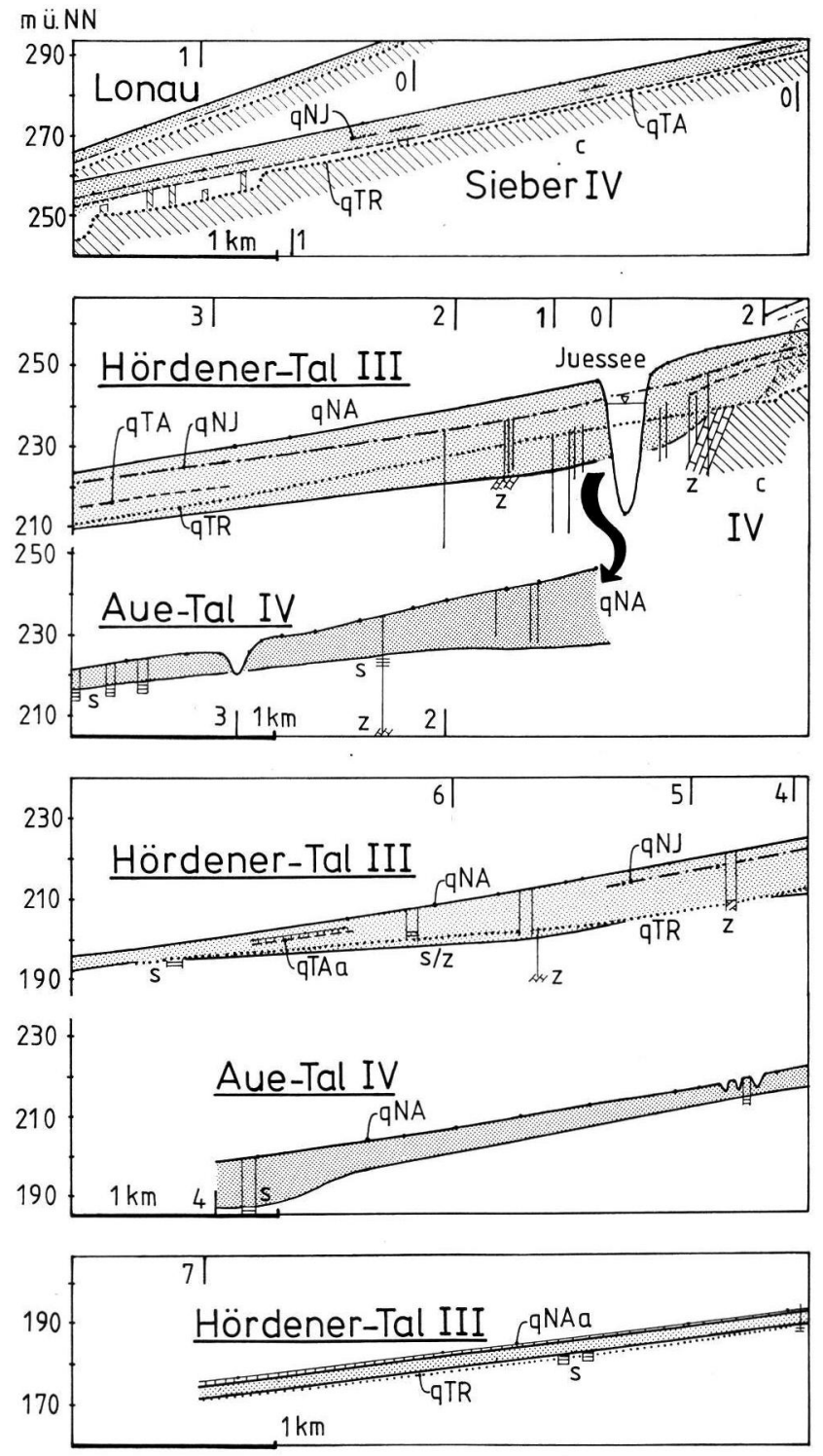

Abb. 8: Kieskörper der Alteren Niederterrasse, Längsprofil durch Hördener Tal (III) und Aue Tal (IV). Profilverlauf, Lage von Bohrungen und Aufschlüssen s. Abb. 1. Bohrungen ergänzt nach Vladi (1976), SEedorf (1955) und Piehler (1969). Hördener Tal und Aue Tal sind getrennt dargestellt, der Bifurkationsbereich ist durch Pfeil gekennzeichnet. Bezeichnungen wie in Abb. 7. Im Hördener Tal sind zusätzlich folgende Erosionsniveaus bzw. Kieskörper angegeben (vgl. Abb. 4): qNJ: Jüngere Niederterrasse. qTA: Ältere Talaue. qTJ: Jüngere Talaue. qTR: Rezente Talaue. Bedeckung von Terrassen mit Auelehm (a): Leiter-Signatur. 
und Lonau flossen also bei maximaler Eintiefung vor der

Sedimentation der Niederterrasse weitgehend im Niveau ihrer späteren Kiesbasis.

Nach diesen Befunden wurde das A u e-Tal irgendwann zwischen dem Ende der Saale-Zeit und dem Altweichsel, also i m Eem-Interglazial, zugunsten des Hördener-Tales aufgegeben und dieses um den doppelten Betrag wie das Auetal eingetieft. In diesem übertieften Talabschnitt wurde dann im Altweichsel der mächtige Kieskörper der Niederterrasse sedimentiert, wobei die Tal-Rinne während der Akkumulation wahrscheinlich durch Seitenerosion erweitert wurde. Durch die rasche Aufsedimentation - die Aue-Berg-Terrasse II (Altweichsel) liegt nur geringfügig über dem Niveau der Alteren Niederterrasse - w urde das A ue-Tal schon früh, d.h. während des Altweichsels, wieder in die Sedimentation mit e in bezogen.

Abgesehen von dem ungewöhnlich frühen Trockenfallen des Aue-Tales zu Beginn des Spätglazials verhält sich die heutige, holozäne Sieber ähnlich wie die Eem-Sieber: Sie tieft sich in der Subrosionssenke bei Herzberg enorm ein, nämlich maximal $18 \mathrm{~m}$ (!) gegenüber der Alteren Niederterrasse (Abb. 8); das ist das 6fache der Eintiefung in den Buntsandstein-Gebieten. Wie im Eem-Interglazial bilden die Sieber und ihre Nebenbäche Gr. Steinau, Gr. Lonau, Gr. Gründelke, verursacht durch die rückschreitende Erosion, Steilgefälle am Harzrand aus; die Sieber und die beiden letztgenannten Bäche haben klammartige Schluchten in Grauwacken und Terrassen-Kiesen (z. B. Lonauer Wasserfall).

Nach dem derzeitigen Kenntnisstand können die Phasen mit Bifurkation bzw. nicht Bifurkation der Sieber gemäß Tabelle 2 festgelegt werden.

Tab. 2: Abriß der Flußgeschichte der Sieber (zeitliche Einordnung der Kieskörper nach Tab. 1)

\begin{tabular}{|c|c|c|}
\hline & Hördener-Tal (subsequent) & Aue-Tal (konsequent) \\
\hline Holozän & $\begin{array}{l}\text { Erosion u. Sedimentation } \\
\text { wechselnd }\end{array}$ & trocken \\
\hline Weichsel-Glazial & mehrphasige Kiessedimentation & mehrphasige Kiessedimentation \\
\hline Eem-Interglazial & überwiegend Erosion & zunächst noch Erosion, dann trocken \\
\hline Warthe-Stadial oder älter & einphasige Kiessedimentation & cinphasige Kiessedimentation \\
\hline $\begin{array}{l}\text { Drenthe/Warthe- } \\
\text { Interstadial ? }\end{array}$ & kräftige Erosion & $\begin{array}{l}\text { Erosion, } \\
\text { Trockenfallen möglich }\end{array}$ \\
\hline Drenthe-Stadial ? & Kiessedimentation (?) & Kiessedimentation \\
\hline Holstein-Interglazial ? & kräftige Erosion & kräftige Erosion \\
\hline Elster-Glazial ? & Kiessedimentation & Kiessedimentation wahrscheinlich \\
\hline
\end{tabular}

\subsection{Klimatische Bedingungen der fluviatilen Akkumulation und Erosion}

Die oben dargelegten Befunde über den Aufbau der Kieskörper und den mit diesen gleichzeitig gebildeten und teilweise überlagernden Lössen und ihre Derivate zeigen, daß fluviatile Akkumulation und Erosion jeweils unter sehr ver$s \mathrm{ch}$ i edenen Klimabedingungen stattfinden können. Da die Kiese der Oberterrasse und Alteren Mittelterrasse ungenügend aufgeschlossen sind, erfolgt die Interpretation vor allem an der Mittelterrasse, Niederterrasse und jüngeren Bildungen. 


\subsubsection{Akkumulation}

\subsubsection{Akkumulation unter kalt-feuchten Klimaverhältnissen}

Ein Beispiel hierfür stellt die in Subrosionsgebieten seh $\mathrm{r}$ mä chtige Ablagerung des unteren Kieskörpers der Alteren Niederterrasse dar, der sich am Aue-Berg sehr wahrscheinlich in der Aue-Berg-Terrasse II fortsetzt. Wie oben dargelegt, fällt die Sedimentation der Kiese in das Altweichsel, einen in sich gegliederten Abschnitt von rd. 30000 Jahren, in dem sich am SW-Harz boreale Nadel- und Birkenwälder und waldfreie Phasen abwechselten. Wie die statistische Auswertung von Lößbohrungen ergeben hat, war das Altweichseldie Periode mit dergrößten solifluidalen Umla g e r u n g innerhalb der letzten Eiszeit (Ricken, im Druck), (Abb. 6). So sind Altweichsel-zeitliche Umlagerungssedimente aus resedimentierten Terrassen-Kiesen und umgelagerten Parabraunerde-Pseudogleyen am SW-Harz weit verbreitet, wie z. B. die Schwemmfächer auf der Aue-Berg-Terrasse I. Da in dieser Periode der s ol if 1 u id a le Sedimentumsatz a ßerordentlich hoch war, andererseits genügend Lockermaterial aus der überwiegend chemischen Verwitterungsperiode des Eem-Interglazials zur Verfügung stand, sedimentierten die Flüsse einen mächtigen mit braunem Lehmmaterial durchsetzten Kieskörper.

Möglicherweise gehören die bis $100 \mathrm{~m}$ mächtigen Kiese der Oberterrasse in der Subrosionssenke bei Osterode ebenfalls in diese Kategorie. JoRdan (1976) beschreibt, daß die Kiese stark von Solifluktionsmaterial durchsetzt sind. Schlufflinsen aus dem unteren Teil enthalten Kohlen von Pinus sp. (JoRdan 1976).

\subsubsection{Akkumulation unter arktischen Klimaverhältnissen}

Hierzu gehören der größte Teil des Kieskörpers der Mittelterrasse, welcher nach den überdeckenden Lößböden wahrscheinlich synchron mit der Warthe-zeitlichen Löß-Akkumulation sedimentiert wurde, möglicherweise aber doch noch älter ist (RICKEN, im Druck). Ebenso ist der obere Kieskörper der Alteren Niederterrasse, der den Jungweichsel-Löß faziell vertritt, als eine Ablagerung in hochglazialem Klima aufzufassen. Beiden Kieskörpern ist gemeinsam, daß sie nur we n ig L e h m mate rial enthalten und deshalb „sauber" erscheinen. Die mittlere Korngröße schwankt zwischen Feinkies und Grobkies; demnach waren die Hochwässer wechselnd stark. Für die Bereitstellung von Gesteinsschutt unter hochglazialen Klimabedingungen kommen im wesentlichen nur die Bildung von Frostschutt und Moränenmaterial aus der Eigenvergletscherung des Harzes (DUPHORN 1969), nicht aber Solifluktion in Frage. Nach Abb. 6 war die Solifluktion im oberen Jungweichsel äußerst gering, trotz gelegentlicher schwacher Bodenbildung bei oberflächlich getautem Dauerfrostboden.

\subsubsection{Akkumulation unter warmzeitlichen Klimaverhältnissen}

Geringmächtige Akkumulation von nur Feinkiesen und Sanden in den jungholozänen Talauen der Flüsse am Südharz zeigen die geringe Wirkung holozäner Hochwässer, trotz weitgehender Entwaldung des Südwestharzes als Folge der Holzkohlengewinnung im 17. und 18. Jahrhundert.

\subsubsection{Erosion}

\subsubsection{Erosion unter kalten bis arktischen Klimaverhältnissen}

Verschiedentlich wurde während kleinerer Erosionsphasen in der Weichsel-Zeit offenbar so langsam eingetieft, daß es nicht zur Bevorzugung von wenigen Rinnen kam, auf denen Abfluß und Erosion verstärkt erfolgen konnten. Vielmehr wird der Habitus des

9 Eiszeitalter u. Gegenwart 
anastomosierenden Flußlaufes beibehalten (vgl. Rinnensystem im Aue-Tal, Abb. 4), und der Aktivitätsbereich nur bei kleineren Flüssen erheblich eingeschränkt (Markau). Beispiele hierfür sind die Erosionsphasen nach Akkumulation der Aue-Berg-Terrasse II, die Eintiefungsphasen von Sieber und Markau im frühen Jungweichsel und die Eintiefung der Jüngeren Niederterrasse im Spätweichsel. Während dieser Phasen wurden die Flußauen zwar nur wenige Meter eingetieft, die Volumenbeträge sind aber wegen der Breite der Eintiefung hoch. Klimatisch sind diese Abschnitte durch $\mathrm{Tun} \mathrm{d}$ r a b öd e n und eine fehlende oder geringe Lößsedimentation gekennzeichnet (Abb. 6). Ein Dauerfrostboden fehlte, oder war oberflächlich getaut, wie im Spätweichsel und während der Bildungsphasen von Herzberger Boden und dem wj 1-Bodenkomplex. Offenbar sind, als Folgen leichter Klimaverbesserungen, erhöhte Abflüsse aufgetreten, wie die Verhältnisse im Reliktlauf des Aue-Tales nahelegen.

$\mathrm{Zu}$ bedeutender kaltklimatisch geprägter Erosion in den Buntsandsteingebieten von gut $25 \mathrm{~m}$ Tiefe kam es nach der Kiesakkumulation der Alteren Mittelterrasse, eine richtige Korrelation von Lößböden und Terrassen vorausgesetzt. Die Erosion fand offenbar im Drenthe/Warthe-Interstadial statt, wo es nach den Befunden der Lößstratigraphie lediglich neben geringer Löß-Sedimentation zur Bildung von kryoklastisch geprägten Tundraböden kam (Hördener Bodenkomplex, Ricken, im Druck).

\subsubsection{Erosion unter warmzeitlichen Klimaverhältnissen}

Das vorliegende Beobachtungsmaterial reicht vorläufig nicht aus, um den Zeitpunkt der Erosion in den Interglazialen einzuengen. Es ist nicht sicher, ob die Erosion schon unter kaltklimatischen Bedingungen im frühen oder erst im späten Interglazial oder während des Wärmemaximums erfolgte. Nach den Terrassen-stratigraphischen Befunden ist im Holozän die Erosion außerhalb der Herzberger Subrosionssenke nach Tiefe, vor allem aber nach Volumen gering. Läßt man die jungholozänen, anthropogen gebildeten Feinkieskörper unberücksichtigt, so ergeben sich für die holozäne Eintiefung ab der Jüngeren Niederterrasse Beträge von $2 \mathrm{~m}$ bis $3 \mathrm{~m}$. Die hohen Eintiefungs-Beträge, die für das Spätweichsel und das Holozän der Abb. 6 entnommen werden können, sind durch Auslaugungsvorgänge verstärkt und gelten nur für die Subrosionssenke bei Herzberg.

\section{Fluviatile Subrosionssenken}

Im südharzer Zechsteingürtel und in den durch Salzauslaugung entstandenen Subrosionsbecken in S-Niedersachsen wurden Quartärfüllungen bis zu $100 \mathrm{~m}$ und darüber beobachtet (z. B. Benda et al. 1968; BrunotTe et al. 1977; Jordan 1976, 1979; Kriebel 1969; LÜTTIG 1969; SeEdORF 1951; STREIF 1970), wobei es sich um genetisch verschiedene Typen von Subrosionssenken handelt. Die hier näher erläuterten fluviatilen Subrosionssenken besitzen im Vergleich zu limnischen Subrosionsbecken wegen der Vorgänge von Akkumulation, Erosion und Seitenerosion eine Eigendynamik. Einfache Beziehungen zwischen Sedimentfüllung und Absenkungsbetrag, wie sie bei limnischen Subrosionsbecken im Idealfall angewandt werden können, bestehen nicht.

\subsection{Synsedimentäre Subrosion}

Das ca. $3 \mathrm{~km}$ breite und $5 \mathrm{~km}$ lange Kieslager der Niederterrasse der Oder im Bereich von Pöhlde, das sogenannte "Pöhlder Becken “ (Abb. 1) kann als eine typische fluviatile Subrosionssenke mit synsedimentärer Subrosion gelten. Das Pöhlder Becken ist über einem Horst im Hauptanhydrit angelegt, der gegen den umgebenen Buntsandstein versetzt ist. Das Verwerfungssystem hat etwa $100 \mathrm{~m}$ Sprunghöhe und verläuft in mehreren Linien nordöstlich vom Rotenberg (JoRDAN 1979). Der ehemals oberflächennah an- 
stehende Hauptanhydrit wurde fast völlig abgelaugt und im wesentlichen durch Terrassen-Kiese ersetzt. Der untere Kieskörper der Älteren Niederterrasse der Oder ist auf einem rd. $4 \mathrm{~km}$ langen Talstück von normal $5 \mathrm{~m}$ bis $10 \mathrm{~m}$ auf $65 \mathrm{~m}$ verdickt und bildet eine große Kieswanne (Abb. 9 und Jordan 1979, Abb. 2). Eine Stratifizierung des KiesBohrgutes aus den in Abb. 9 angegebenen Wasserbohrungen ist durch den Vergleich mit benachbarten Terrassen-Kiesen möglich. Mittel- und Niederterrassen-Kiese der Oder sind aufgrund ihres Gehaltes an Grauwacken unterscheidbar, da während der Sedimentation der Mittelterrasse die Sieber noch in breiter Front in das Pöhlder Becken mündete und dort den Grauwacken-Anteil der Oder-Kiese erhöhte. Wegen des stark aufgemahlenen Bohrgutes konnten nur noch Kiese der Fraktion $2 \mathrm{~mm}$ bis $4 \mathrm{~mm}$ bestimmt werden (Bruch, Lupe). Die Ergebnisse sind in Tabelle 3 dargestellt.

Gegenüber Aufschlüssen enthält das Bohrgut um 10 bis $20 \%$ erhöhte Gehalte an Tonschiefer, der offenbar beim Bohrvorgang selektiv stark zerkleinert wird und teilweise Gerölle vortäuscht. Bohrproben mit einem Quotienten von Grauwacke zu Tonschiefer von 0,49 bis 0,58 dürften der Niederterrasse zuzurechnen sein, Quotienten von 0,77 bis 0,86 der Mittelterrasse. Der Wert von 0,63 vermittelt zwischen diesen Gruppen. Es könnte sich hierbei um Eem-Kiese handeln.

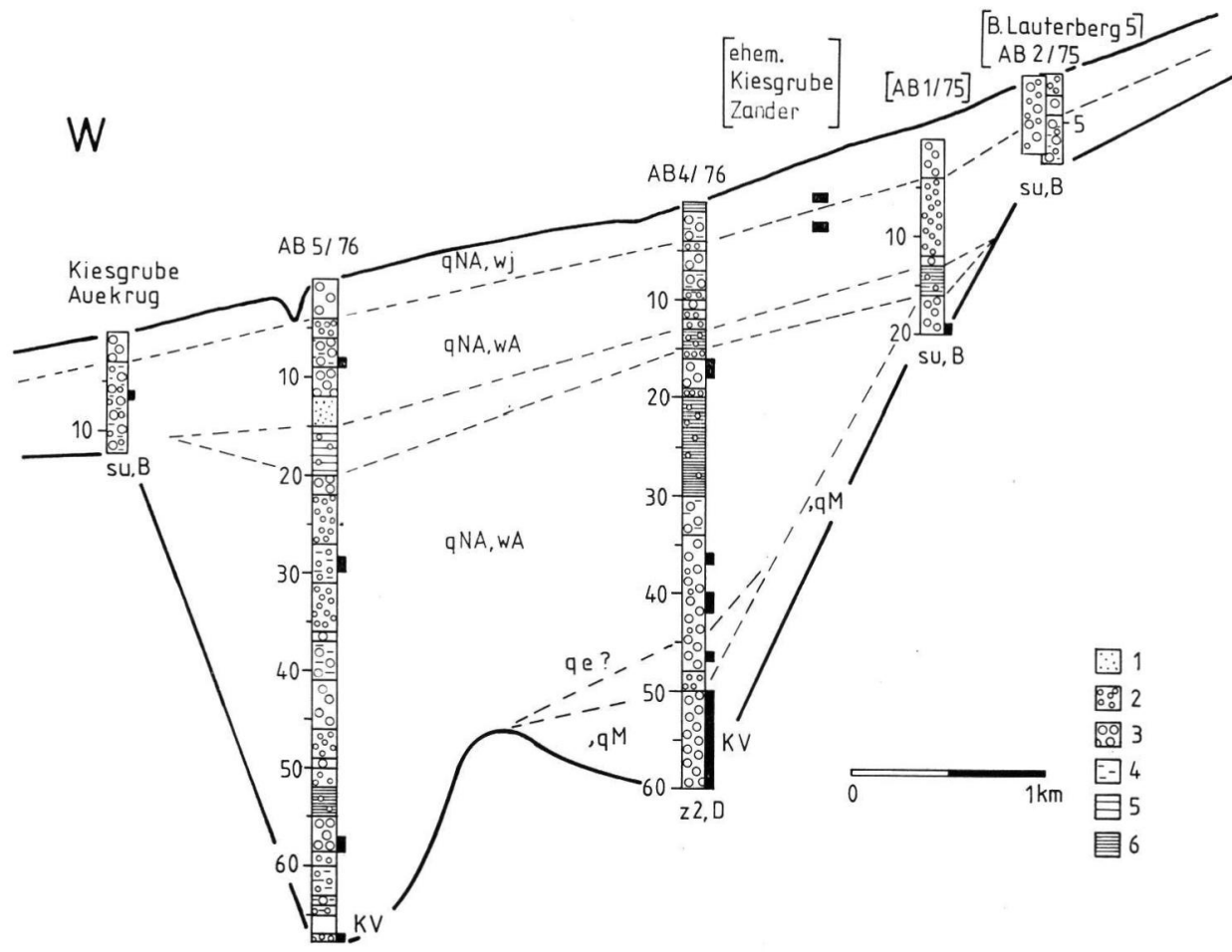

Abb. 9: Längsprofil durch das Pöhlder Becken (V). Lage von Profil, Bohrungen und Aufschlüssen s. Abb. 1. Projezierte Bohrungen und Aufschlüsse in Klammern. Probeentnahme für Geröllzählungen, vgl. Tab. 3. z2, D: Staßfurt-Dolomit des Zechsteins. su, B: Bröckelschiefer des Unteren Buntsandsteins. qM: Kies der Mittelterrasse. qe ?: Kiese fraglich Eem. qNA: Kiese der Alteren Niederterrasse. wj, wa: Jung- und Altweichsel. KV: Kernverlust. 1 Sande, Feinkies. 2 Kies. 3 Gerölle. 4 schwach schluffig. 5 schluffig. 6 stark schluffig bis tonig. 
Tab. 3: Pöhlder Becken, Zusammensetzung von Kiesen der Frakton 2 bis $4 \mathrm{~mm}$ in \% (Bohrungen und Kiesgruben bzw. nat. Aufschlüsse Entnahmestellen der Proben s. Abb. 9.

\begin{tabular}{|c|c|c|c|c|c|c|c|c|c|c|}
\hline $\begin{array}{l}\text { Bohrung } \\
\text { Kiesgrube }\end{array}$ & Meter & $\begin{array}{l}\text { Grund- } \\
\text { menge }\end{array}$ & $\begin{array}{l}\text { Grau- } \\
\text { wacke }\end{array}$ & $\begin{array}{l}\text { Ton- } \\
\text { schiefer }\end{array}$ & $\begin{array}{l}\text { Kiesel- } \\
\text { schiefer }\end{array}$ & $\begin{array}{l}\text { Granit } \\
\text { Hornfels }\end{array}$ & Quarz & Quarzit & Sonstige & $\begin{array}{l}\text { Grauwacke } \\
\text { zu } \\
\text { Tonschiefer }\end{array}$ \\
\hline Zander & $4-5$ & 200 & 29,9 & 55,4 & 2,7 & 4,0 & 7,6 & 0,4 & 0,0 & 0,54 \\
\hline Zander & $7-8$ & 200 & 30,5 & 52,5 & 2,0 & 4,0 & 9,0 & 2,0 & 0,0 & 0,58 \\
\hline Auekrug & $6-7$ & 200 & 31,5 & 44,0 & 5,0 & 3,0 & 16,0 & 0,5 & 0,0 & 0,72 \\
\hline $\mathrm{AB} 5 / 76$ & $28-30$ & 241 & 27,8 & 65,6 & 0,9 & 1,2 & 4,1 & 0,0 & 0,4 & 0,42 \\
\hline $\mathrm{AB} 5 / 76$ & $57-59$ & 248 & 30,7 & 62,9 & 1,2 & 1,2 & 4,0 & 0,0 & 0,0 & 0,49 \\
\hline $\mathrm{AB} 4 / 76$ & $17-18$ & 118 & 30,8 & 60,3 & 3,0 & 4,9 & 1,0 & 0,0 & 0,0 & 0,51 \\
\hline $\mathrm{AB} 4 / 76$ & $40-42$ & 161 & 31,6 & 58,5 & 1,2 & 6,8 & 1,9 & 0,0 & 0,0 & 0,54 \\
\hline $\mathrm{AB} 4 / 76$ & $36-37$ & 166 & 32,2 & 58,4 & 2,4 & 5,4 & 0,6 & 1,0 & 0,0 & 0,55 \\
\hline $\mathrm{AB} 5 / 76$ & $8-9$ & 273 & 32,9 & 58,6 & 2,2 & 4,4 & 1,5 & 0,0 & 0,4 & 0,56 \\
\hline AB 5/76 & $67-68$ & 235 & 31,9 & 54,9 & 1,3 & 5,1 & 6,0 & 0,4 & 0,4 & 0,58 \\
\hline $\mathrm{AB} 4 / 76$ & $46-47$ & 173 & 34,3 & 54,7 & 1,1 & 7,0 & 2,9 & 0,0 & 0,0 & 0,63 \\
\hline $\mathrm{AB} 1 / 75$ & $19-20$ & 173 & 39,3 & 50,9 & 0,6 & 5,2 & 4,0 & 0,0 & 0,0 & 0,77 \\
\hline $\mathrm{AB} 4 / 76$ & $50-60$ & 145 & 40,6 & 47,0 & 1,2 & 9,7 & 1,5 & 0,0 & 0,0 & 0,86 \\
\hline \multicolumn{2}{|c|}{ Mittelterrasse } & 200 & 45,0 & 42,4 & 3,6 & 2,0 & 5,0 & 2,0 & 0,0 & 1,06 \\
\hline
\end{tabular}


Da der verdickte Sedimentkörper überwiegend nicht aus Seesedimenten, sondern aus Kiesen aufgebaut ist, müssen diese aus einem Niveau, in dem die Oder noch kein Gegengefälle bekam, abgesenkt worden sein. Sie sind vermutlich in den liegenden Teilen zunehmend ungeschichtet. $\mathrm{Da}$ jedoch in den Kiesgruben bis zu einer aufgeschlossenen Tiefe von maximal $10 \mathrm{~m}$ oberer und unterer Kieskörper überwiegend geschichtet sind $-\mathrm{z}$. T. mit ausgedehnten Schlufflagen - muß die Absenkung synsedimentär erfolgt sein und zwar mit einer nach oben abnehmenden Intensität.

Wegen der Erweiterung durch Seitenerosion und der Klima-bedingten Akkumulation entspricht der aus dem Volumen der Quartär-Füllung des Pöhlder Beckens errechenbare subrosive Absenkungsbetrag nicht der wahren Absenkung. Bei einer Übertiefung von $40 \mathrm{~m}$ bis $55 \mathrm{~m}$ gegenüber der normalen Mächtigkeit des unteren Kieskörpers von ca. $10 \mathrm{~m}$ und einer vermuteten Sedimentationsdauer für das Altweichsel von rd. 30000 Jahren ergeben sich für den Zentralteil des Pöhlder Beckens Absenkungsbeträge von $13 \mathrm{~m}$ bis $18 \mathrm{~m}$ in 10000 Jahren.

\subsection{Synerosive Subrosion}

In der Subrosionssenke des Werra-Anhydrits, die sich von Herzberg ca. $3 \mathrm{~km} \mathrm{NW}$ in das Siebertal erstreckt, kommt es heute, wie auch früher im Eem-Interglazial, zu einer ausgeprägten Tiefenerosion (vergl. Kapitel 2.7.). Die Eintiefung erreicht gegenüber der Niederterrasse maximal $18 \mathrm{~m}$. Dabei verläuft die Oberfläche der Jüngeren Niederterrasse noch weitgehend parallel mit der Alteren Niederterrasse, während die Talauen-Stufen etwa zwischen Harz und dem Buntsandstein-Ausstrich gegenüber der Niederterrasse durchgebogen sind (Abb. 8). Die Übertiefung in der Subrosionssenke beendete zunächst die Bifurkation der Sieber und verursachte am Harzrand Steilgefälle und Schluchten - eine Folgewirkung der rückschreitenden Erosion. Die Periodizität der Übertiefung des Hördener-Tales und das damit gekoppelte Versiegen des Aue-Tales im Spätweichsel und Holozän, im Eem und vermutlich auch vor der Sedimentation der Mittelterrasse zeigt, $\mathrm{da} ß$ es sich hierbei $\mathrm{n} \mathrm{icht} \mathrm{um} \mathrm{ein} \mathrm{tektonisches} \mathrm{Phänomen} \mathrm{einer} \mathrm{Terrassenverbiegung} \mathrm{im}$ Sinne einer "Harzhebung " handeln kann (HövermanN 1950), zumal die Oberflächen der Kieskörper von Niederterrasse und Mittelterrasse parallel liegen. Ebenso kann nicht in Frage kommen, daß die pleistozänen Kieslager in der Subrosionssenke leichter als die Grauwacken und die Gesteine des Unteren Buntsandsteins erodiert werden. Die Oder fließt nämlich im Pöhlder Becken ebenfalls in mächtigen Kiesen, ohne sich in diese besonders ausgeprägt einzuschneiden. Ein Vergleich der Terrassen an Söse, Sieber und Oder durch Piehler (1969) und die Untersuchungen im Raum Seesen durch Thiem (1972) zeigen, daß kräftige holozäne Eintiefung nur dann aufritt, : wenn unter den übertieften Talabschnitten Gipse liegen. Die Oder quert zwar auch den Zechstein wie andere Harzflüsse. Jedoch wird ihr Kieskörper aufgrund fazieller Änderungen des Zechsteins mit dem Übergang von Anhydrit zu Dolomit und aufgrund der weitgehend abgeschlossenen Ablaugung des Hauptanhydrits (JoRDAN 1979, Abb. 2) im Pöhlder Becken nur noch gering von Gips unmittelbar unterlagert.

Wie sich aus Kiesgruben-Aufschlüssen, Datierungen von Erdfällen und der Auswertung von Erdfall-Böschungen ergibt, fehlt während des Hochglazials am SW-Harz die subrosive Absenkung oder war gering. Sie setzt erst im Spätglazial ein, erreicht insgesamt im Präboreal bis Atlantikum ein Maximum und dauert heute noch an (Ricken 1980: 50). Damit fällt am SW-Harz die postglaziale Subrosion zeitlich mit der postglazialen Eintiefungstendenz der Flüsse zusammen. Dieser Umstand ist wahrscheinlich für die TalUbertiefung verantwortlich. Vermutlich kommt es zu einer Wechselwirkung zwischen Erosions- und Subrosionsvorgängen, die die Intensivie- 
rung beider Prozesse im Talbereich zur Folge hat (synerosive Subrosion). Durch die Eintiefung wird dem liegenden Gipskarst verstärkt sulfatarmes Wasser zugeführt, so daß die Gipsauflösung erhöht wird. Erdfälle und Senken, die sich in der Talaue bilden, tragen zur Tieferlegung des Untergrundes bei. Sie regen die Erosion an, da sie als Sedimentfänger wirken und Steilkanten usw. als Ansatzpunkte für die Erosion schaffen. Als Folge entstehen gegenüber den Normalgebieten übertiefte Talabschnitte, die dann in der nachfolgenden Kaltzeit mit Kiesen verfüllt werden. Auf diese Weise bilden sich übernormal mächtige, aber geschichtete Kieskörper. So ist im Hördener-Tal der Eem-zeitlich übertiefte Talabschnitt (mehrphasig) durch die Sedimentation geschichteter Kiese der Älteren Niederterrasse wieder verfüllt worden. In t e r g l a z i a le Ưbertiefung und (früh)- kaltzeitliche Auffüllung der übertiefte n $\mathrm{T} \ddot{\mathrm{l}} \mathrm{le} \mathrm{r}$, die dabei durch Seitenerosion erweitert werden, ist analog zu den Vorgängen im Sieber-Tal vermutlich eine wesentliche Ursache für die übernormal mächtigen, aber geschichteten Kiesvorkommen im Zechstein-Ausstrich des Südharzes. Hierzu gehören wahrscheinlich die gut $40 \mathrm{~m}$ mächtigen Kiese der Oberterrasse bei Osterode im Bereich des Butter-Berg-Tunnels. Jordan (1976), der zahlreiche Bohrungen und Tiefenaufschlüsse beim Tunnelbau ausgewertet hat, beschreibt diese Kiese als bis zu ihrer Basis geschichtete Ablagerungen mit z. T. $100 \mathrm{~m}$ horizontal durchhaltenden Schlufflinsen, die nur lokal verstellt sind.

Für die Erklärung übernormal mächtiger geschichteter Kieslager scheint eine großflächige, synsedimentäre Absenkung von Kiesen im Verband nur eine untergeordnete Rolle zu spielen. Erstens spricht die Situation im Sieber-Tal gegen eine solche Deutung, wo geschichtete mächtige Kiese noch auf Harz-Paläozoikum liegen. Zweitens haben aktuogeologische Beobachtungen an 290 ungestörten Erdfällen und Subrosionssenken auf verschiedenen Flächen der Niederterrasse des Süd-Harzes ergeben, daß Erdfälle des Spätweichsels und Holozäns 7 mal häufiger sind als flache, durch subrosive Absenkungen entstandene Senken und Erdfälle $5 \mathrm{mal}$ höhere Subrosionsvolumina als diese aufweisen.

\section{Dank}

Herr Prof. B. Meyer (Inst. f. Bodenkunde, Göttingen), der auch das Manuskript redigierte, förderte die Arbeit durch sein andauerndes Interesse und seine tatkräftige Hilfe bei Gelände- und Laborarbeiten. Herr Dr. Grüger (Abt. f. Palynologie, Göttigen) und Herr Prof. Willerding (Göttingen) übernahmen die Paläobotanischen Bestimmungen. Herr Dr. Нонм (Geol. Inst. Hannover) schoß mit seiner Hammerschlag-Seismik ein $120 \mathrm{~m}$ langes Querprofil durch das Aue-Tal. Herr Dr. Jordan (Nieders. L. A. f. Bodenf.) vermittelte ein ${ }^{14} \mathrm{C}$-Datierung und Bohrgut aus den Wasserbohrungen im Pöhlder Becken. Herr Prof. Semmel (Geogr. Inst. Frankfurt), Herr Dr. Hannss (Geogr. Inst., Tübingen), Herr Prof. Meischner und Herr Dr. Paul (beide Geol.-Paläont.-Inst., Göttingen) verdanke ich wichtige Literaturhinweise und kritische Stellungnahmen. Herr Prof. EINSELE (Geol.-Paläont.-Inst., Tübingen) sah ein frühes Manuskript dieser Arbeit kritisch durch.

Bei der Bohr-Arbeit im Gelände halfen: Rainer BicK (Göttingen), Michael JAHN (Northeim), Christian Miehr (Berlin), Hildburg Stöckmann (Han. Münden), Dipl. Geol. Firouz Vladi (Osterode), Hajo Weinberg (Hamburg) und Hilmar Zander (Berlin). 


\section{Schriftenverzeichnis}

Behre, K. E. (1974): Die Vegetation im Spätpleistozän von Osterwanna/Niedersachsen. - Geol. Jb., A 18: 3-48, 8 Abb., 5 Taf.; Hannover.

Benda, L., Gaertner von, H. R., Herrmann, R., Lüttig, G., Streif, H., Vinken, R. \& WunderLICH, H. G. (1968): Känozoische Sedimente in tektonischen Fallen und Subrosionssenken in Süd-Niedersachsen. - Z. dt. geol. Ges., 117: 713-726, 1 Abb., 1 Tab., Hannover.

BRunotTe, E. (1978): Zur quartären Formung von Schichtkämmen und Fußflächen im Bereich des Markoldendorfer Beckens und seiner Umrahmung. - Göttinger geograph. Abh., 72: 1-138, 51 Abb., 6 Tab., 4 Beil.; Göttingen.

- \& Sickenberg, O. (1977): Die mittel- und jungquartäre Entwicklung des Leinetales zwischen Northeim und Salzderhelden unter besonderer Berücksichtigung der Subrosion. - Geol. Jb., A 44: 3-43, 14 Abb., 4 Tab., 4 Taf.; Hannover.

Duphorn, K. (1968): Ist der Oberharz im Pleistozän vergletschert gewesen? - Eiszeitalter u. Gegenwart, 19: 164-174, 4 Abb.; Óhringen.

- (1969): Geologische Ergebnisse einer Grabung in der Einhornhöhle bei Scharzfeld am Harz. - Jahresh. Karst u. Höhlenkde., 9: 83-90, 2 Abb.; München.

FINK, J. (1962): Studien zur absoluten und relativen Chronologie der fossilen Böden in Ósterreich. II Wetzleinsdorf und Stillfried. - Archacologia Austriaca, 31: 1-18, 7 Abb.; Wien.

HaAse, H., Schmidt, M. \& Lenz, J. (1970): Der Wasserhaushalt des Westharzes (Hydrologische Untersuchungen 1941-1965). - Veröff. d. nieders. Institut für Landesk. u. Landesentw. an d. Univers. Göttingen, A 95: 1-96, 59 Abb., 27 Taf., 133 Tab.; Göttingen.

Hillegeist, H. H. (1977): Die Geschichte der Lonauer Hammerhütte bei Herzberg/Harz. 193 S., 75 Abb., 1 Kt.; Göttingen (Vandenhoeck \& Ruprecht).

Hövermann, J. (1949): Morphologische Untersuchungen im Mittelharz. - Göttinger geograph. Abh., 2: 1-80, 8 Taf., 1 Kt.; Göttingen.

- (1950): Die diluvialen Terrassen des Oberharzes und seines Vorlandes. Ein Beitrag zur Frage der Harzhebung. - Peterm. geogrph. Mitt., 3: 121-130, 9 Abb., 1 Kt.; Gotha/Leipzig.

Jordan, H. (1976): Die Terrassenkiese im Sösetal am Harz. — Geol. Jb., A 36: 75-101, 13 Abb., 1 Tab.; Hannover.

- (1979): Der Zechstein zwischen Osterode und Duderstadt (südliches Harzvorland). - Z. dt. geol. Ges., 130: 145-163, 5 Abb., 8 Tab.; Hannover.

Kriebel, U. (1969): Die Wechselbeziehungen zwischen Abtragung, Subrosion und Tektonik im südlichen Harzvorland. - Geologie, 18: 638-650, 9 Abb.; Berlin.

Kulick, J. \& SEmmel, A. (1968): Die geomorphologische und geologische Bedeutung der Paläolith-Station Buhlen (Waldeck). - Notizbl. Hess. Landesamt Bodenforsch., 76: 347-351, 2 Abb., 1 Taf.; Wiesbaden.

Löscher, M., Bruns, M., Hieronymus, U., Mäusbacher, R., Münnich, M., Münzig, K. \& Schedler, J. (1980): Neue Ergebnisse über das Jungquartär im Neckarschwemmfächer bei Heidelberg. - Eiszeitalter u. Gegenwart, 30: 89-100, 3 Abb., 4 Tab., 1 Taf.; Hannover.

LüтtiG, G. (1960): Neue Ergebnisse quartärgeologischer Forschung im Raum Alfeld-HamelnElze. - Geol. Jb., 77: 337-390, 11 Abb., 3 Taf., 5 Tab.; Hannover.

- (1969): Abnorme Quartärprofile im nordwestdeutschen Bergland. - Geol. Jb., 88: 13-34, 5 Abb., 2 Tab.; Hannover.

Mania, D. \& Töpfer, V. (1973): Königsaue. Gliederung, Okologie und mittelpaläolithische Funde der letzten Eiszeit. Teil III Die Vollgliederung des letzten Klimazyklus nach der Sedimentserie aus dem Ascherslebener See (Profil Königsaue). - Veröff. Landesmus. Vorgesch. Halle, 26: 1-162, 14 Abb., 73 Taf.; Berlin (Deut. Verl. Wiss.).

Munsell (1954): Soil color charts. - Munsell Color Company; Baltimore.

Piehler, U. (1969): Die Täler der Oder, Sieber und Söse und das Problem der Harzhebung. Staatsexamensarb. Geographie Univers. Göttingen: 1—79, 19 Abb.; Göttingen. — [Unveröff.] 
RohDEnBURG, H. (1966): Eiskeilhorizonte in südniedersächsischen und nordhessischen Lößprofilen. — Mitt. dt. bodenkundl. Ges., 5: 137-170, 20 Abb.; Göttingen.

- \& Merer, B. (1966): Zur Feinstratigraphie und Paläopedologie des Jungpleistozäns nach Untersuchungen an südniedersächsischen und nordhessischen Lößprofilen. - Mitt. dt. bodenkl. Ges., 5: 1-137, 25 Abb.; Göttingen.

RICKEN, W. (1973): Zur Rekonstruktion der geologischen Verhältnisse im Pleistozän bei Herzberg (Südwestharz) mit Hilfe der Schotteranalyse. - Jahresarbeit Kreisgymnasium Herzberg: 1-59, 19 Abb., 19 Tab., 1 Kt.; Herzberg. - [Unveröff.]

- (1980): Quartäre fluviatile und äolische Sedimentation am Südwest-Harz und ihre Beeinflussung durch die Subrosion. - Dipl.-Arbeit Geol.-Paläont.-Inst. Göttingen: 1-71, Anhang, 40 Abb., 7 Tab., 2 Kt.; Göttingen. - [Unveröff.]

- (1982): Mittel- und jungpleistozäne Lößdecken im südwestlichen Harzvorland. Stratigraphie, Paläopedologie, fazielle Differenzierung und Konnektierung mit Flußterrassen. - in: BoRK, H. R. \& RICKEN, W.: Bodenerosion, holozäne und pleistozäne Bodenentwicklung. - Catena Supplement 3; Cremlingen. - [Im Druck].

Rössert, R. (1976): Hydraulik im Wasserbau. - 180 S., 149 Abb., 25 Tab.; München (R. Oldenbourg Verlag, 3. Aufl.).

Scheer, H. D. (1978): Gliederung und Aufbau der Niederterrassen von Rhein und Main im nördlichen Oberrheingraben. - Geol. Jb. Hessen, 106: 273-289, 4 Abb.; Wiesbaden.

Schönhals, E., Rohdenburg, H. \& Semmel, A. (1964): Ergebnisse neuerer Untersuchungen zur Würm-Gliederung in Hessen. - Eiszeitalter u. Gegenwart, 15: 199-206, 1 Abb.; Öhringen.

Sснӥтt, G. (1968): Die cromerzeitlichen Bären aus der Einhornhöhle bei Scharzfeld. - Mitteil. geol. Inst. T. H. Hannover, $7: 1-120$; Hannover.

SeEdorf, H. H. (1955): Reliefbildung durch Gips und Salz im niedersächsischen Bergland. Nieders. Amt f. Landespl. u. Statistik, A 1, 56: 1-109, 41 Abb., 4 Kt.; Bremen/Horn.

Seidenschwann, G. (1980): Zur pleistozänen Entwicklung des Main-Kinzig-Kahl-Gebietes. Rhein-Mainische Forsch., 91: 1-194, 18 Abb., 2 Tab., 1 Kt.; Frankfurt/Main.

Semmel, A. (1968): Studien über den Verlauf jungpleistozäner Formung in Hessen. - Frankfurter Geogr. Hefte, 45: 1-133, 35 Abb.; Frankfurt/Main.

- (1972): Untersuchungen zur jungpleistozänen Talentwicklung in deutschen Mittelgebirgen. Z. Geomorphol. N. F., Suppl. Bd., 14: 105-112, 3 Abb., Berlin/Stuttgart.

- (1974): Der Stand der Eiszeitforschung im Rhein-Main-Gebiet. — in: Semmel, A. Hrg. (1974): Das Eiszeitalter im Rhein-Main-Gebiet. - Rhein-Mainische-Forschungen, 78: 9-56, 5 Abb.; Frankfurt/Main.

- \& StÄBlEIN, G. (1971): Die Entwicklung quartärer Hohlformen in Franken. - Eiszeitalter u. Gegenwart, 22: 23-34, 6 Abb.; Óhringen.

Simons, D. B., Richardson, E. V. \& Guy, H. P. (1966): Summary of alluvial channel data from flume experiments 1961-1965. - U. S. Geol. Survey Professional Paper, 462 -I: 1-96; Washington.

StEInmüller, A. (1965): Eine weichselzeitliche Schichtenfolge in der goldenen Aue bei Nordhausen. - Jb. Geologie, 1: 373-394, 13 Abb., 4 Tab.; Berlin.

StreIf, H. (1970): Limnologische Untersuchung des Seeburger Sees (Untereichsfeld). Geol. Untersuchungen an Niedersächsischen Binnengewässern VII. - Beih. Geol. Jb., 83: 1-106, 25 Abb., 9 Taf.; Hannover.

ThIEM, W. (1972): Geomorphologie des westlichen Harzrandes und seiner Fußregion. - Jahrb. geogr. Gesell. Hannover, Sonderh., 6: 1-271, 35 Abb., 19 Taf.; Hannover.

UNGER, K. (1974): Quartär. in: Hoppe, A. \& SeIdel, G. Hrg. (1974): Geologie von Thüringen. 742-781, 17 Abb., 14 Tab.; Gotha/Leipzig (VEB Herrmann Haack).

VLADI, F. (1976): Quartärgeologische Untersuchungen zu den Terrassen der Sieber am Südwestrande des Harzes. - Dipl.-Arbeit Geol.-Paläont.-Inst. Univers. Hamburg: 1-109, 14 Abb., Anhang Ktn., Profile, Tab.; Hamburg. - [Unveröff.]

Wildhagen, H. \& Meyer, B. (1972): Holozäne Boden-Entwicklung, Sediment-Bildung und Geomorphogenese im Flußauen-Bereich des Göttinger Leinetal-Grabens. - Göttinger Bodenkl. Ber., 21: 1-158, 15 Abb., 9 Kt., 10 Tab.; Göttingen.

Wijmstra, T. A. (1978): Palaeobotany and climatic change. in: Gribbin, J. Hrg. Climatic change. - 25-45, 6 Abb.; Cambridge (University Press).

Manuskript eingegangen am 23.11. 1981. 